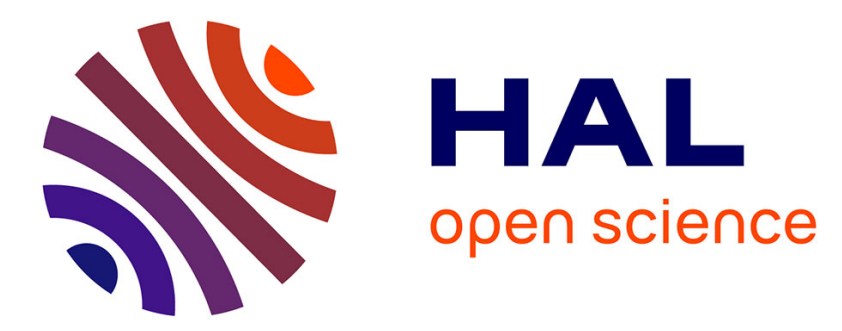

\title{
Feedback control of the general two-trailers system with the Transverse Function approach
}

\author{
Pascal Morin, Claude Samson
}

\section{To cite this version:}

Pascal Morin, Claude Samson. Feedback control of the general two-trailers system with the Transverse Function approach. [Research Report] RR-7938, INRIA. 2012, pp.27. hal-00690532

HAL Id: hal-00690532

https://inria.hal.science/hal-00690532

Submitted on 23 Apr 2012

HAL is a multi-disciplinary open access archive for the deposit and dissemination of scientific research documents, whether they are published or not. The documents may come from teaching and research institutions in France or abroad, or from public or private research centers.
L'archive ouverte pluridisciplinaire HAL, est destinée au dépôt et à la diffusion de documents scientifiques de niveau recherche, publiés ou non, émanant des établissements d'enseignement et de recherche français ou étrangers, des laboratoires publics ou privés. 


\section{Feedback control of the} general two-trailers system with the Transverse Function approach

Pascal Morin, Claude Samson

RESEARCH REPORT

$\mathbf{N}^{\circ} \mathbf{7 9 3 8}$

Avril 2012

Project-Team Arobas 



\title{
Iñía
}

\section{Feedback control of the general two-trailers system with the Transverse Function approach}

\author{
Pascal Morin *, Claude Samson \\ Project-Team Arobas \\ Research Report n 7938 - Avril 2012 - 27pages
}

\begin{abstract}
The so-called "general two-trailers system" is a nonholonomic system composed of a controlled unicycle-like vehicle and two passive trailers with off-axle hitching. It is not differentially flat and cannot be transformed into the chained form system. Methods developed for this latter class of systems thus do not apply. The Transverse Function (TF) approach is here used to solve the trajectory tracking problem for this system. The proposed control solution yields practical stabilization of any reference motion, whether it is or is not feasible. Practical stabilization of nonfeasible trajectories in the case of non-differently flat systems is of particular interest due partly to the difficulty of planning and calculating desired feasible state reference motions. The method is illustrated by simulation results which show that, in addition to the unconditional practical stabilization property evoked above, asymptotic stabilization of feasible and persistently exciting motions can also be achieved with the same performance as local stabilizers derived from a linear approximation of the tracking-error equations.
\end{abstract}

Key-words: General trailer system, nonholonomic system, transverse function, trajectory tracking

* This author is with ISIR-UPMC, Paris, France.

\section{RESEARCH CENTRE}

SOPHIA ANTIPOLIS - MÉDITERRANÉE

2004 route des Lucioles - BP 93

06902 Sophia Antipolis Cedex 


\section{Research report Inria}

Résumé : Le "système général à deux remorques" est un système non-holonome composé d'un véhicule commandé de type unicycle, et de deux remorques passives dont le point d'attache est déporté par rapport à l'axe des roues du véhicule précédent. Ce système n'est pas différentiellement plat, et ne peut donc pas être transformé en un système chainé. Les méthodes de commande développées pour cette dernière classe de systèmes ne s'appliquent donc pas au système général à deux remorques. Dans ce rapport, l'approche de commande par fonctions transverses est appliquée à ce système pour résoudre le problème de stabilisation de trajectoire. La commande proposée assure la stabilisation pratique de trajectoires quelconques, i.e. qu'elles soient réalisables par le système ou non. Cette propriété est particulièrement intéressante pour les systèmes non différentiellement plats, sachant qu'il est difficile dans ce cas de déterminer explicitement des trajectoires de référence réalisables. La méthode proposée est validée par des résultats de simulation illustrant, outre la propriété de stabilisation pratique évoquée précédemment, la possibilité de stabiliser asymptotiquement les trajectoires de référence réalisables et satisfaisant la propriété classique dite "d'excitation persistante", avec des performances comparables à celles obtenues avec une commande linéaire synthétisée à partir d'une approximation linéaire du système d'erreur.

Mots-clés : Système général à remorques, système non-holonome, fonction transverse, stabilisation de trajectoire 


\section{Contents}

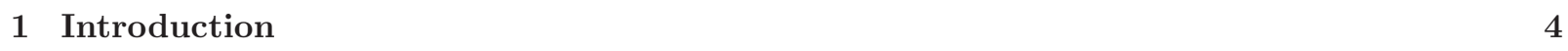

2 Notation and recalls 5

2.1 Svstems on Lie groups . . . . . . . . . . . . . . . . . . . . . . . . . . . 5

2.2 Transverse Functions $\ldots \ldots \ldots \ldots \ldots \ldots$

\begin{tabular}{|lll|}
\hline & Modeling and properties of the control Lie algebra & 6
\end{tabular}

3.1 Modeling . . . . . . . . . . . . . . . . . . . . . . . 7

3.2 Properties of the control Lie algebra $\ldots \ldots \ldots \ldots \ldots \ldots$

\begin{tabular}{|lr|r}
\hline 4 Control design & 8
\end{tabular}

4.1 Control objective . . . . . . . . . . . . . . . . . . . . . . . . . . . . 8

4.2 Extension to a svstem on a Lie group $\ldots \ldots \ldots \ldots$

4.3 Transverse functions $\ldots \ldots \ldots \ldots \ldots \ldots \ldots$

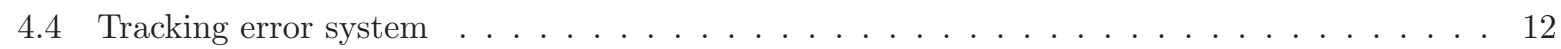

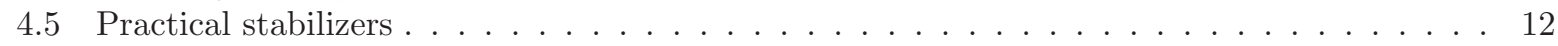

4.6 Asvmptotic stabilization of reference trajectories with constant curvature $\ldots \ldots \ldots$. . . . . 13

$4.7 \quad$ Linear asymptotic stabilizers of reference trajectories with constant curvature $\ldots \ldots \ldots$

$\begin{array}{lll}5 \text { Simulation results } & 15\end{array}$

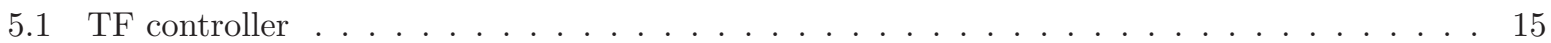

5.2 Linear controllen . . . . . . . . . . . . . . . . . . . . . . . . . . . 17

$\begin{array}{llr}6 & \text { Conclusion } & 18\end{array}$

\begin{tabular}{ll}
\hline References & 27
\end{tabular}

$\begin{array}{ll}\text { Appendix 1: Proof of Theorem 2 } & 27\end{array}$

Appendix 2: Proof of Proposition 2 27 


\section{Introduction}

Nonlinear control of the so-called " $N$-trailers system" (i.e. a unicycle-like vehicle with $N$ trailers) has motivated numerous studies during the past two decades. Most of them assume that each trailer is hitched at a point on the wheels' axle of the preceding vehicle. This assumption implies that the system's kinematic model can be transformed into the canonical chained form, as shown by Murray and Sastry [1] in the one-trailer case, and by Sørdalen [2] in the case of an arbitrary number of trailers. The wealth of stabilization techniques developed for control systems in the chained form can then be applied. Another consequence of this property is that the system is differentially flat 3 . This allows for straightforward solutions to the path planning problem consisting essentially in the calculation of "feasible" reference trajectories, i.e. state trajectories that satisfy the system's motion equations, passing through desired state configurations. While a unicycle, or car, with a single off-axle hitched trailer remains differentially flat [4, this property is lost when two or more trailers are hitched off axle. This latter case is usually referred to as the "general trailer system". The general two-trailers system, corresponding to a unicycle vehicle with two off-axle hitched trailers, is thus the simplest example in this category. The fact that, to our knowledge, no exact path planner has ever been proposed for this type of systems is much related to the system's non-flatness. Although this difficulty seems, at first glance, disconnected from the trajectory tracking problem, it is in fact one of the motivations of the present study. The reason is that the trajectory tracking problem, commonly defined as the problem of stabilizing a reference trajectory asymptotically, i.e. with convergence of the tracking error to zero in addition to stability in the sense of Lyapunov, is well posed only when the reference trajectory is feasible. The difficulty of designing feasible reference trajectories between arbitrary state configurations led, in the past, trajectory tracking methods for the general trailer system to focus on very specific feasible reference trajectories, like fixed-points [5, 6] and circular-like trajectories [5]. The present paper takes a different route. It is based on the Transverse Function (TF) approach developed by the authors in the last decade (see e.g [7, [8]) with the prime objective of achieving the practical stabilization of any-feasible or non-feasible- reference motion. The above considerations explain why bypassing the feasibility issue is of particular interest for systems which are not differentially flat. Now, practical stabilization of any reference trajectory is by no means opposed to asymptotic stabilization of feasible and persistently exciting reference trajectories, which should rather be considered as a complementary, more demanding and restritive, objective. The present study shows how these two objectives can be combined in a single feedback control law. However, by lack of a complete proof, the zeroing of the tracking errors is only illustrated here through simulation results.

The paper is related to several previous works by the authors, and more particularly to 9 where the control of a three-segments wheeled snake-like mechanism is addressed. The kinematic model equations of this wheeled mechanism and of the general two-trailers system are in fact identical. However, due to different means of actuation -shape angles actuation vs. wheels actuation- their control properties are different. In particular, some geometric configurations, such as aligned vehicles, are singular -and thus must be avoided- for the snake mechanism, whereas they are not singular for the trailer system. On the other hand, local controllability at these configurations, i.e. the satisfaction of the Lie Algebra Rank Condition (LARC), involves Lie brackets of order three whereas it is sufficient to go to order two when the trailers are not aligned [9]. This issue is related to singularities of the control Lie algebra [10] which, in the snake-mechanism's case, coincide with actuation singularities, and the passage through which is avoided by forbidding all wheels' axles to intersect at the same point (which can be at infinity). Undulatory snakelike locomotion stems from this constraint. By contrast, in the trailer's case such configurations are not singular w.r.t. the actuation and thus should be permitted, all the more so that they allow for longitudinal motion without energy consuming maneuvers. However, due to the Lie algebra singularity issue evoked previously, achieving the practical stabilization of any reference trajectory turns out to be more difficult for the general two-trailers system. This fact also explains the chronology followed by the authors for the studies of these two systems. With respect to [9], the present study shows how to deal with singular configurations in order to best exploit the motion capabilities of the trailer system and, in doing so, how to overcome the superior complexity induced by the necessity of using Lie brackets of higher order in the 
control solution.

The paper is organized as follows. Preliminary technical recalls and notation are provided in Section 2. The system's kinematic model and some of its control properties are presented in Section 3 . The main contribution, which details the control design methodology based on the application of the TF approach, is presented in Section 4. Comparative simulation results of the TF control solution and a controller derived from a linear approximation of the system are reported in Section 5 . Finally, the concluding Section 6 points out future research directions.

\section{Notation and recalls}

In this paper, $x^{\prime}$ denotes the transpose of a vector $x \in \mathbb{R}^{n}, I_{n}$ is the identity matrix of dimension $(n \times n)$, and $O_{m \times n}$ is the zero-valued matrix of dimension $(m \times n) . \mathbb{T}^{p}$ denotes the $p$-dimensional torus.

\subsection{Systems on Lie groups}

Only basic properties of systems on Lie groups will be used. A few definitions and notation are recalled hereafter. The reader is referred, e.g., to [1] for more details in the context of the control of nonholonomic systems.

The tangent space of a manifold $M$ at a point $q$ is denoted as $T_{q} M$. If $X$ is a vector field (v.f.) on $M$, the solution at time $t$ of $\dot{x}=X(x)$ with initial condition $x(0)=q$ is denoted as $\exp (t X, q)$. A Lie group $G$ is a manifold with a group operation $\left(g_{1}, g_{2}\right) \longmapsto g_{1} g_{2}$ such that the mapping $\left(g_{1}, g_{2}\right) \longmapsto g_{1} g_{2}^{-1}$ is smooth, with $g^{-1}$ denoting the group inverse of $g$. Let $G$ denote a connected Lie group of dimension $n$. The unit element of $G$ is denoted as $e$, i.e. $\forall g \in G, g e=e g=g$. The left and right translation operators on $G$ are denoted as $L$ and $R$ respectively, i.e. $\forall(\sigma, \tau) \in G^{2}, L_{\sigma}(\tau)=R_{\tau}(\sigma)=\sigma \tau$. A v.f. $X$ on $G$ is left-invariant iff $\forall(\sigma, \tau) \in G^{2}, d L_{\sigma}(\tau) X(\tau)=X(\sigma \tau)$, with $d f$ denoting the differential of a function $f$. The Lie algebra -of left-invariant v.f.- of the group $G$ is denoted as $\mathfrak{g}$. If $X \in \mathfrak{g}, \exp (t X)$ is used as a short notation of $\exp (t X, e)$. A driftless control system $\dot{g}=\sum_{i=1}^{m} X_{i}(g) \xi_{i}$ on $G$ is said to be left-invariant on $G$ if the control v.f. $X_{i}$ are left-invariant. With $f, g$, and $h$ denoting smooth curves on $G$, one has (omitting the time index)

$$
\frac{d}{d t}\left(g f^{-1}\right)=d R_{f^{-1}}(g)\left(\dot{g}-d L_{g f^{-1}}(f) \dot{f}\right)
$$

and

$$
\frac{d}{d t}\left(h^{-1} g\right)=d L_{h^{-1}}(g) \dot{g}-d R_{h^{-1} g}(e) d L_{h^{-1}}(h) \dot{h}
$$

In the special case of the Lie group $G=\mathbb{S E}(2)$, the group operation is defined by

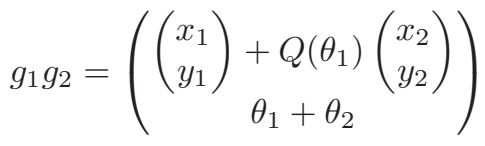

with $g_{i}=\left(x_{i}, y_{i}, \theta_{i}\right)^{\prime}$ and $Q(\theta)$ the rotation matrix in the plane of angle $\theta$. The unit element is $e=(0,0,0)^{\prime}$ and the inverse of $g=(x, y, \theta)^{\prime}$ is

$$
g^{-1}=\left(\begin{array}{c}
-Q(-\theta)\left(\begin{array}{l}
x \\
y
\end{array}\right) \\
-\theta
\end{array}\right)
$$

One deduces from (3) that

$$
d L_{g_{1}}\left(g_{2}\right)=\left(\begin{array}{cc}
Q\left(\theta_{1}\right) & 0_{2 \times 1} \\
0_{1 \times 2} & 1
\end{array}\right)
$$

and

$$
d R_{g_{2}}\left(g_{1}\right)=\left(\begin{array}{cc}
I_{2} & Q\left(\theta_{1}\right)\left(\begin{array}{c}
-y_{2} \\
y_{1}
\end{array}\right) \\
0_{1 \times 2} & 1
\end{array}\right)
$$

RR n 7938 
The family $\mathbf{X}=\left\{X_{1}, X_{2}, X_{3}\right\}$ of v.f. defined by $X_{i}(g)=X(g) e_{i}, i=1,2,3$ with

$$
X(g)=\bar{Q}(\theta):=\left(\begin{array}{cc}
Q(\theta) & 0_{2 \times 1} \\
0_{1 \times 2} & 1
\end{array}\right)
$$

and $e_{1}, e_{2}, e_{3}$ the canonical basis vectors of $\mathbb{R}^{3}$, constitutes a basis of left-invariant vector fields.

\subsection{Transverse Functions}

Notions about transverse functions are recalled -see e.g. 8] for more details. Let $\mathbf{X}=\left\{X_{1}, \ldots, X_{m}\right\}$ denote a family of smooth v.f. $X_{1}, \ldots, X_{m}$ on a $n$-dimensional manifold $M$. X satisfies the Lie Algebra Rank Condition (LARC) at some point $q_{0}$ if $\operatorname{Lie}(\mathbf{X})\left(q_{0}\right)=T_{q_{0}} M$ with

$$
\operatorname{Lie}(\mathbf{X})=\operatorname{span}\left\{X_{i},\left[X_{i}, X_{j}\right],\left[X_{i},\left[X_{j}, X_{k}\right], \ldots ; i, j, k, \ldots=1, \ldots, m\right\}\right.
$$

and $\operatorname{Lie}(\mathbf{X})(q)=\{X(q): X \in \operatorname{Lie}(\mathbf{X})\}$. Given a compact manifold $K$, a smooth function $f: K \longrightarrow M$ is transverse to $\mathbf{X}$ if, for any $\alpha \in K$,

$$
\operatorname{span}\left\{X_{1}(f(\alpha)), \ldots, X_{m}(f(\alpha)), d f(\alpha)\left(T_{\alpha} K\right)\right\}=T_{f(\alpha)} M
$$

with $d f$ the differential of $f$. Note that the dimension of $K$ must be at least equal to $(n-m)$. Given $q_{0} \in M$ such that the family $\mathbf{X}$ satisfies the LARC at $q_{0}$, the "Transverse Function theorem" in [7] ensures the existence of a family $\left(f^{\varepsilon}\right)_{\varepsilon>0}$ of functions transverse to $\mathbf{X}$, with $\max _{\alpha} \operatorname{dist}\left(f^{\varepsilon}(\alpha), q_{0}\right) \rightarrow 0$ as $\varepsilon \rightarrow 0$, where "dist" denotes any distance locally defined in the neighborhood of $q_{0}$.

We slightly generalize the definition of a transverse function to any smooth function $f: K \times \mathbb{R} \longrightarrow M$ by requiring that for any fixed time value $t$, the function $\alpha \longmapsto f(\alpha, t)$ is transverse in the above sense.

\section{Modeling and properties of the control Lie algebra}

The general two-trailers system, whose view from above is sketched in Fig. 1, is composed of a unicycle-like vehicle pulling two trailers with off-axle hitch points. The control inputs are the unicycle linear velocity $v_{\ell}^{0}$ and its angular velocity $\omega^{0}$. The two trailers are passive. For simplicity, for $i=1,2$ the distance between the center $P_{i}$ of trailer $i$ and its hitching point $L_{i}$, and the distance between $L_{i}$ and $P_{i-1}$ are set equal to one.
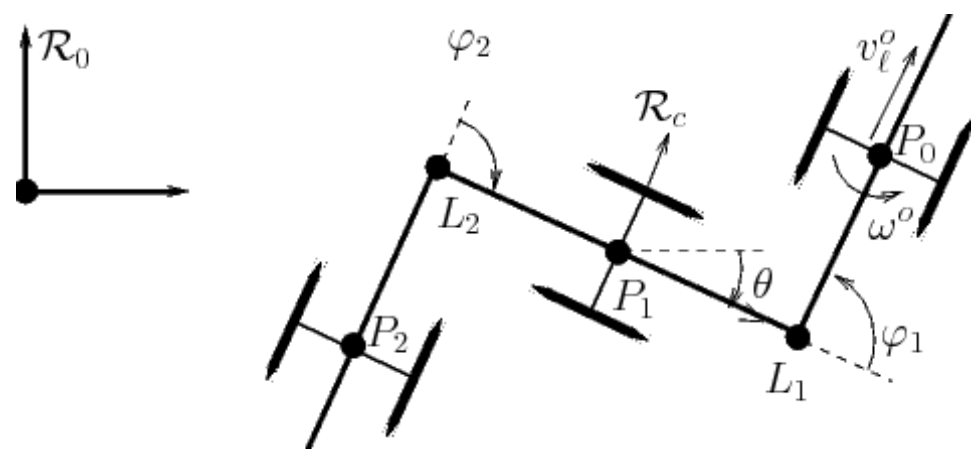

Figure 1: General two-trailer system 


\subsection{Modeling}

For symmetry reasons, a body-fixed frame $\mathcal{R}_{c}$ with origin $P_{1}$ is attached to the mid-vehicle, i.e. the first trailer. The configuration of this vehicle with respect to an inertial frame $\mathcal{R}_{0}$ is thus characterized by

$$
g=\left(\begin{array}{l}
x \\
y \\
\theta
\end{array}\right) \in \mathbb{S E}(2)
$$

with $x$ and $y$ the coordinates of $P_{1}$ in $\mathcal{R}_{0}$, and $\theta$ the orientation of $\mathcal{R}_{c}$ w.r.t. $\mathcal{R}_{0}$. The complete configuration vector of the system is given by $(g, \varphi) \in \mathbb{S E}(2) \times \mathbb{T}^{2}$ with

$$
\varphi=\left(\begin{array}{l}
\varphi_{1} \\
\varphi_{2}
\end{array}\right) \in \mathbb{T}^{2}
$$

the vector of hitch angles, also called the "shape vector" of the system. Let $v_{\ell}^{1}$ and $\omega^{1}$ denote the linear and angular velocities of the first trailer. Then

$$
\left\{\begin{array}{l}
\dot{x}=v_{\ell}^{1} \cos \theta \\
\dot{y}=v_{\ell}^{1} \sin \theta \\
\dot{\theta}=\omega^{1}
\end{array}\right.
$$

From now on, $v=\left(v_{\ell}^{1}, \omega^{1}\right)^{\prime}$ will be the considered as the vector of control inputs. It is related to the unicycle's velocity vector $\left(v_{\ell}^{0}, v_{\theta}^{0}\right)^{\prime}$ according to the biunivocal transformation

$$
v=Q\left(\varphi_{1}\right)\left(\begin{array}{c}
v_{\ell}^{0} \\
-\omega^{0}
\end{array}\right)
$$

The classical non-slipping assumption associated with wheel-ground contact, applied to both trailers, yields the following two nonholonomic constraints:

$$
\begin{aligned}
& -v_{\ell}^{1} \sin \varphi_{1}+\omega^{1}\left(1+\cos \varphi_{1}\right)+\dot{\varphi}_{1}=0 \\
& v_{\ell}^{1} \sin \varphi_{2}-\omega^{1}\left(1+\cos \varphi_{2}\right)+\dot{\varphi}_{2}=0
\end{aligned}
$$

In matrix form these equations can be written as

$$
\dot{\varphi}=A_{\varphi}(\varphi) v
$$

with

$$
A_{\varphi}(\varphi)=\left(\begin{array}{cc}
\sin \varphi_{1} & -1-\cos \varphi_{1} \\
-\sin \varphi_{2} & 1+\cos \varphi_{2}
\end{array}\right)
$$

Eq. (9]10) define a kinematic control system for the trailer system with $v$ as the control input. The following change of coordinates

$$
\varphi \longmapsto \eta=\left(\begin{array}{l}
\eta_{1} \\
\eta_{2}
\end{array}\right)=\left(\begin{array}{c}
\tan \frac{\varphi_{1}}{2} \\
\tan \frac{\varphi_{2}}{2}
\end{array}\right)
$$

is well defined away from the "jack-knife" angles $\varphi_{1,2}=\pi$ and is useful to avoid these "bad" hitch angle values. It also simplifies the control system equations. Indeed, from (9]10) one has

$$
\dot{\eta}=A(\eta) v, \quad A(\eta)=\left(\begin{array}{cc}
\eta_{1} & -1 \\
-\eta_{2} & 1
\end{array}\right)
$$

so that each shape variable $\eta_{i}$ now satisfies a polynomial differential equation. By regrouping relations (9) and (13) one obtains the following driftless control model :

$$
\left\{\begin{array}{l}
\dot{g}=X(g) C v \\
\dot{\eta}=A(\eta) v
\end{array}\right.
$$

$\mathrm{RR} \mathrm{n}^{\circ} 7938$ 
with $X(g)$ defined by (7) and

$$
C=\left(\begin{array}{ll}
1 & 0 \\
0 & 0 \\
0 & 1
\end{array}\right)
$$

Setting $\xi=\left(g^{\prime}, \eta^{\prime}\right)^{\prime}$, this system may also be written as

$$
\dot{\xi}=Z_{1}(\xi) v_{1}+Z_{2} v_{2}
$$

with

$$
Z_{1}(\xi)=\left(\begin{array}{c}
\cos \xi_{3} \\
\sin \xi_{3} \\
0 \\
\xi_{4} \\
-\xi_{5}
\end{array}\right), Z_{2}=\left(\begin{array}{c}
0 \\
0 \\
1 \\
-1 \\
1
\end{array}\right)
$$

\subsection{Properties of the control Lie algebra}

The following proposition recalls controllability properties of System (16) and establishes other properties which play a key role in the forthcoming control design.

Proposition 1. The family of v.f. $\mathbf{Z}=\left\{Z_{1}, Z_{2}\right\}$ satisfies the following properties:

1. $\operatorname{dim} \operatorname{Lie}(\mathbf{Z})(\xi)=\operatorname{dim}(\xi)$ for any $\xi$, i.e. the LARC is satisfied at any point.

2. With $Z_{3}=\left[Z_{1}, Z_{2}\right], Z_{4}=\left[Z_{1}, Z_{3}\right], Z_{5}=\left[Z_{2}, Z_{3}\right], Z_{6}=\left[Z_{1}, Z_{4}\right]$, and $Z_{7}=\left[Z_{2}, Z_{5}\right]$, one has

$$
\begin{aligned}
\operatorname{Lie}(\mathbf{Z}) & =\operatorname{span}_{\mathbb{R}}\left\{Z_{1}, \ldots, Z_{5}, Z_{6}\right\} \\
& =\operatorname{span}_{\mathbb{R}}\left\{Z_{1}, \ldots, Z_{5}, Z_{7}\right\}
\end{aligned}
$$

3. $\left[Z_{1}, Z_{5}\right]=\left[Z_{2}, Z_{4}\right]=0$.

Proof: The proof follows by a direct calculation of the Lie brackets of $Z_{1}, Z_{2}$ up to the fourth order.

\section{Control design}

\subsection{Control objective}

Given a (any) reference Cartesian motion for the trailer system, specified by a reference trajectory $g_{r}()=$. $\left(x_{r}, y_{r}, \theta_{r}\right)^{\prime}(.) \in \mathbb{S E}(2)$, one of the control objectives is to stabilize at zero the tracking error defined by

$$
\tilde{g}=g_{r}^{-1} g
$$

More precisely, the objective is to achieve the practical stabilization of $\tilde{g}=0$, i.e. the stabilization of a set inside a given neighborhood of $\tilde{g}=0$, having in mind that the more demanding objective of asymptotic stabilization of $\tilde{g}=0$ is possible only when the reference trajectory $g_{r}$ is feasible for the mid-vehicle. In view of (14) this latter constraint implies that the reference frame lateral velocity, i.e. the second component of the vector $v_{r}$ defined by $\dot{g}_{r}=X\left(g_{r}\right) v_{r}$, is identically equal to zero. In addition, the control law must ensure the boundedness of the internal shape vector $\eta$ so as to prevent the unicycle and its trailers from colliding into each other, a phenomenom known as the "knife-edge" effect. Respecting this constraint constitutes in fact the real challenge, since otherwise it would suffice to control one of the vehicles without paying attention to the others. Another (complementary) objective is to achieve the asymptotic stabilization of feasible trajectories, i.e. the asymptotic stabilization of $\tilde{g}=0$. This property implies that $v$ converges 
asymptotically to $v_{r}$ and that along the reference trajectory $\eta$ satisfies the following relation (compare with (13) )

$$
\dot{\eta}=A(\eta)\left(v_{r, 1}, v_{r, 3}\right)^{\prime}
$$

For arbitrary time functions $v_{r, 1}, v_{r, 3}$, this system of equations does not admit closed-form solutions. This raises the difficulty of specifying a reference value $\eta_{r}$ for $\eta$. Note, however, that

$$
\eta_{r, 1}=\eta_{r, 2}=c_{r}:=\frac{v_{r, 3}}{v_{r, 1}}
$$

is a solution when the ratio $v_{r, 3} / v_{r, 1}$ is constant. This case corresponds to motions along straight lines and circles, with $c_{r}$ the associated curvature. Provided that the linearized system along these trajectories is controllable - a property easily established when $v_{r, 1}(t)>\epsilon>0$ - one can also apply classical linear control techniques to stabilize such trajectories. For the sake of completeness, a control law of this type is derived in Section 5 and its performance compared in simulation with the more complex, but also more versatile, TF control solution here proposed.

\subsection{Extension to a system on a Lie group}

Although System (16) is not a system on a Lie group, Property 2 of Proposition 1 points out that the Lie algebra generated by $Z_{1}, Z_{2}$ is finite-dimensional, of dimension equal to six. This implies that there is a Lie group associated with this Lie algebra and that the v.f. $Z_{1}, Z_{2}$ can be "lifted" to left-invariant v.f. on a 6-dimensional manifold. This property, which to our knowledge has not been reported before in the literature, will be exploited further for the control design.

Theorem 1. Consider the dynamic extension of (16) defined by

$$
\dot{\bar{\xi}}=\bar{Z}_{1}(\bar{\xi}) v_{1}+\bar{Z}_{2} v_{2}
$$

with

$$
\bar{\xi}=\left(\begin{array}{c}
\xi \\
\bar{\xi}_{6}
\end{array}\right), \bar{Z}_{1}(\bar{\xi})=\left(\begin{array}{c}
Z_{1}(\xi) \\
1
\end{array}\right), \bar{Z}_{2}=\left(\begin{array}{c}
Z_{2} \\
0
\end{array}\right)
$$

Then,

1. $\overline{\mathbf{Z}}=\left\{\bar{Z}_{1}, \bar{Z}_{2}\right\}$ satisfies Properties 1,2 , and 3 of Proposition 1 .

2. The v.f. $\bar{Z}_{1}, \bar{Z}_{2}$ are left-invariant on $\mathbb{S E}(2) \times \mathbb{R}^{3}$ with respect to the group operation defined by

$$
x \bullet y=\left(\begin{array}{c}
g_{x} g_{y} \\
x_{4} \exp \left(y_{6}\right)+y_{4} \\
x_{5} \exp \left(-y_{6}\right)+y_{5} \\
x_{6}+y_{6}
\end{array}\right)
$$

with $g_{x}$ and $g_{y}$ denoting the first three components of $x$ and $y$ respectively.

3. The differentials of the left and right translation operators associated with the group operation (22) are given by

$$
d L_{x}(y)=\left(\begin{array}{ccc}
\bar{Q}\left(x_{3}\right) & & O_{3 \times 3} \\
O_{3 \times 3} & \left(\begin{array}{ccc}
1 & 0 & x_{4} \exp \left(y_{6}\right) \\
0 & 1 & -x_{5} \exp \left(-y_{6}\right) \\
0 & 0 & 1
\end{array}\right)
\end{array}\right)
$$

and

$$
d R_{y}(x)=\left(\begin{array}{cc}
d R_{1,1}\left(x_{3}, y_{1,2}\right) & O_{3 \times 3} \\
O_{3 \times 3} & d R_{2,2}\left(y_{6}\right)
\end{array}\right)
$$

$\mathrm{RR} \mathrm{n}^{\circ} 7938$ 
with

$$
d R_{1,1}\left(x_{3}, y_{1,2}\right)=\left(\begin{array}{cc}
I_{2} & Q\left(x_{3}\right)\left(\begin{array}{c}
-y_{2} \\
y_{1}
\end{array}\right) \\
0_{2 \times 1} & 1
\end{array}\right), \quad d R_{2,2}\left(y_{6}\right)=\left(\begin{array}{ccc}
\exp \left(y_{6}\right) & 0 & 0 \\
0 & \exp \left(-y_{6}\right) & 0 \\
0 & 0 & 1
\end{array}\right)
$$

Proof: The proof consists of basic calculations.

Remark and correction of [12, Prop. 2]: Except for the Lie group invariance property, the control v.f. of System (21) share the same properties as the control v.f. of System (16). In particular, one verifies that the linearization of System (21) along feasible reference trajectories -for which $v_{r, 2} \equiv 0$ - associated with constant reference velocities $v_{r_{1}}$ and $v_{r_{3}}$ is also controllable when $v_{r, 1} \neq 0$. This property constitutes a counter-example to an earlier result, namely [12, Prop. 2], which points out conditions on the structure of a control Lie algebra that are necessary to the controllability of a linear approximation along feasible trajectories generated with constant control inputs. It also gives us the opportunity to indicate that the proof of this result is correct when a complementary assumption of homogeneity upon the control v.f. is made. Since the control v.f. of (21) are not homogeneous w.r.t. any dilation, by the simple fact that they involve trigonometric functions, the above mentioned result does not apply and the controllability of the linear approximation cannot be ruled out.

\subsection{Transverse functions}

Previous papers, like 8 and 13 for instance, point out general algorithms for the determination of transverse functions (TF) that work for any set of control v.f. which satisfy the LARC. However, given a physical system and various TF for this system, no systematic methodology exists at this time to $a$ priori select a "good" candidate in terms of some performance criterion, itself not easily defined in the case of systems performing maneuvers. Let us just mention the sensible rule -reflecting the authors' own experience in this matter- according to which the design of TF should make use of the geometrical properties of the system's control vector fields. For instance, while the functions proposed in 8 are defined on a torus, we showed more recently in [14] and [13. how to define TF on a special orthogonal group when the LARC is satisfied with first-order Lie brackets only. This latter possibility is preferable to the former one for systems that exhibit specific symmetry properties (see [13, Sec. III] for more details). More generally, it is possible to combine different definition domains so as to produce TF defined on a product of toruses and special orthogonal groups. The TF proposed next, for a class of families of v.f. which includes the family $\left\{\bar{Z}_{1}, \bar{Z}_{2}\right\}$ of System (21), belong to this third category.

The following notation will be used.

1. $\Delta_{\varepsilon}$ and $P$ denote constant matrices:

$$
\Delta_{\varepsilon}=\operatorname{Diag}\left(\varepsilon, \varepsilon, \varepsilon^{2}, \varepsilon^{3}, \varepsilon^{3}\right), \quad P=\left(\begin{array}{ccc}
0 & -1 & 0 \\
1 & 0 & 0
\end{array}\right)
$$

with $\operatorname{Diag}\left(x_{1}, \ldots, x_{p}\right)$ the diagonal matrix whose ordered elements on the diagonal are $x_{1}, \ldots, x_{p}$.

2. Vector-valued functions involved in the calculation of the proposed TF are

$$
\mu_{a_{1}}\left(\theta_{1}\right)=\left(\begin{array}{c}
a_{1,1} s \theta_{1} \\
a_{1,2} c \theta_{1} \\
0 \\
\frac{a_{1,1}^{2} a_{1,2}}{3} c \theta_{1} \\
-\frac{a_{1,1} a_{1,2}^{2}}{3} s \theta_{1}
\end{array}\right), \delta_{a_{2}, a_{3}}\left(\theta_{2}, \theta_{3}\right)=\left(\begin{array}{c}
a_{2,1} s \theta_{2} \\
a_{3,1} s \theta_{3} \\
0 \\
a_{2,2} c \theta_{2} \\
a_{3,2} c \theta_{3}
\end{array}\right), \nu_{D}(Q)=\left(\begin{array}{c}
D Q e_{1} \\
P \bar{D} Q e_{3}
\end{array}\right)
$$

with $Q \in \mathbb{S O}(3), \theta_{i} \in \mathbb{S}^{1}, s \theta_{i}$ and $c \theta_{i}$ standing for $\sin \theta_{i}$ and $\cos \theta_{i}$ respectively, $a_{i}=\left(a_{i, 1}, a_{i, 2}\right)$, and

$$
D=\operatorname{Diag}\left(d_{1}, d_{2}, d_{3}\right), \quad \bar{D}=\operatorname{Diag}\left(d_{2} d_{3}, d_{1} d_{3}, d_{1} d_{2}\right)
$$


3. Given v.f. $X_{1}, \ldots, X_{p}$ and a vector $v \in \mathbb{R}^{p}, X v$ is used for $\sum_{i=1}^{p} v_{i} X_{i}$ to shorten the notation. The following result is obtained by combining the TF theorems in [8] and [13].

Theorem 2. Consider a Lie group $G$ with Lie algebra $\mathfrak{g}$ and denote by $x \bullet y$ the group product of $x$ and $y$ in $G$. Consider two left-invariant v.f. $X_{1}, X_{2} \in \mathfrak{g}$ such that

$$
\mathfrak{g}=\operatorname{Lie}\left(X_{1}, X_{2}\right)=\operatorname{span}_{\mathbb{R}}\left\{X_{1}, \ldots, X_{7}\right\}
$$

and

$$
\left[X_{1}, X_{5}\right],\left[X_{2}, X_{4}\right] \in \operatorname{span}_{\mathbb{R}}\left\{X_{1}, \ldots, X_{5}\right\}
$$

with

$$
X_{3}=\left[X_{1}, X_{2}\right], X_{4}=\left[X_{1}, X_{3}\right], X_{5}=\left[X_{2}, X_{3}\right], X_{6}=\left[X_{1}, X_{4}\right], X_{7}=\left[X_{2}, X_{5}\right]
$$

There exist $\bar{\varepsilon}>0$ and constant parameters $a_{1,2,3}$ and $d_{1,2,3}$ such that, for any $\varepsilon \in(0, \bar{\varepsilon})$, the function $f^{\varepsilon}$ defined on $\mathbb{T}^{3} \times \mathbb{S O}(3)$ by

$$
\begin{gathered}
f^{\varepsilon}\left(\theta_{1}, \theta_{2}, \theta_{3}, Q\right)=f_{3}^{\varepsilon}\left(\theta_{2}, \theta_{3}\right) \bullet f_{2}^{\varepsilon}(Q) \bullet f_{1}^{\varepsilon}\left(\theta_{1}\right) \\
\text { with } \\
f_{1}^{\varepsilon}\left(\theta_{1}\right)=\exp \left(X \Delta_{\varepsilon} \mu_{a_{1}}\left(\theta_{1}\right)\right) \\
f_{2}^{\varepsilon}(Q)=\exp \left(X \Delta_{\varepsilon} \nu_{D}(Q)\right) \\
f_{3}^{\varepsilon}\left(\theta_{2}, \theta_{3}\right)=\exp \left(X \Delta_{\varepsilon} \delta_{a_{2}, a_{3}}\left(\theta_{2}, \theta_{3}\right)\right)
\end{gathered}
$$

is transverse to $X_{1}, X_{2}$.

The proof is given in the appendix.

This result calls for several remarks.

1. From (24), the dimension of $G$ is less than, or equal to, seven. When $\operatorname{dim}(G)<7$, some parameters of the TF can be set equal to zero. For example, if $\mathfrak{g}=\operatorname{span}_{\mathbb{R}}\left\{X_{1}, \ldots, X_{6}\right\}$, one can set $a_{3}=0$, and if $\mathfrak{g}=\operatorname{span}_{\mathbb{R}}\left\{X_{1}, \ldots, X_{5}\right\}$, one can set $a_{2}=a_{3}=0$ so that $f^{\varepsilon}=f_{2}^{\varepsilon}(Q) \bullet f_{1}^{\varepsilon}\left(\theta_{1}\right)$.

2. The rationale behind the proposed TF expressions is as follows. Three functions are involved, namely $f_{1}^{\varepsilon}, f_{2}^{\varepsilon}$, and $f_{3}^{\varepsilon}$. The role of $f_{1}^{\varepsilon}$ is to grant transversality in the direction of the first-order Lie bracket $X_{3}=\left[X_{1}, X_{2}\right]$. More precisely, we have $\dot{f}_{1}^{\varepsilon}=X\left(f_{1}^{\varepsilon}\right) \eta_{\varepsilon, a_{1}}\left(\theta_{1}\right) \dot{\theta}_{1}$ with the third component of $\eta_{\varepsilon, a_{1}}$ -i.e. the coefficient of $X_{3}\left(f_{1}^{\varepsilon}\right)$ - being always different from zero. Similarly, $f_{2}^{\varepsilon}$ grants transversality in the directions of the second-order Lie brackets $X_{4}=\left[X_{1}, X_{3}\right]$ and $X_{5}=\left[X_{2}, X_{3}\right]$, and $f_{3}^{\varepsilon}$ grants transversality in the direction of the third-order Lie brackets $X_{6}=\left[X_{1}, X_{4}\right], X_{7}=\left[X_{2}, X_{5}\right]$, with $\theta_{2}$ yielding the $X_{6}$ direction and $\theta_{3}$ the $X_{7}$ direction.

3. Let $G=\mathbb{S E}(2) \times \mathbb{R}^{3}$. By Theorem $1, \bar{Z}_{1}$ and $\bar{Z}_{2}$ are left invariant v.f. of $G$ and satisfy the properties of Proposition 1. Therefore, they also satisfy the assumptions of Theorem 2, so that TF for $\left\{\bar{Z}_{1}, \bar{Z}_{2}\right\}$ are given by (27). Since $\bar{Z}_{1}, \bar{Z}_{2}$ satisfy Property 2 of Proposition 1, it follows from the above remark that one can set $a_{3}$ (or $a_{2}$ equal to zero). In other words, the dependence of $f_{3}^{\varepsilon}$ (and subsequently $f^{\varepsilon}$ ) on two variables $\theta_{2}$ and $\theta_{3}$, rather than only one -either one of them-, is not completely necessary. It can be interesting to use non-zero values for both pairs of parameters because there is no reason to favor one of the Lie brackets $X_{6}$ and $X_{7}$ w.r.t the other. We have also verified that this redundancy grants more freedom as for the determination of the parameters $a_{1,2,3}$ for which the transversality property holds.

4. Once given the family of functions (27), it remains to determine values of the parameters $\varepsilon, a_{1-3}$ and $d_{1-3}$-i.e. ten scalars- for which the transversality property is satisfied. The number of parameters can be brought back to three because the proof of Theorem 2 can be worked out with $a_{1,1}=a_{1,2}=d_{j}$ $(j=1,2,3), a_{2,1}=a_{3,1}$, and $a_{2,2}=a_{3,2}=a_{2,1}^{3}$. However, using a larger number of parameters yields more flexibility in the control performance and the monitoring of the tracking errors. This can also be useful for the control of other systems that satisfy the assumptions of Theorem 2, For the simulation results reported further on, and in the absence of a better method to characterize a "good" set, we have essentially applied a brute "guess and check" method which allowed us to find out values for which we are reasonably confident -after testing on various feasible and non-feasible trajectories- that they yield transversality and acceptable overall performance of the controller.

$\mathrm{RR} \mathrm{n}^{\circ} 7938$ 


\subsection{Tracking error system}

From (2), (5), (6), (14), and (18), it follows that

$$
\left\{\begin{array}{l}
\dot{\tilde{g}}=X(\tilde{g}) C v+p_{g}\left(\tilde{g}, v_{r}\right) \\
\dot{\eta}=A(\eta) v
\end{array}\right.
$$

with

$$
p_{g}\left(\tilde{g}, v_{r}\right)=-\left(\begin{array}{ccc}
1 & 0 & -\tilde{g}_{2} \\
0 & 1 & \tilde{g}_{1} \\
0 & 0 & 1
\end{array}\right) v_{r}
$$

the additive "perturbation" arising from the motion of the reference frame. Setting now

$$
\xi=\left(\begin{array}{c}
\tilde{g} \\
\eta
\end{array}\right)
$$

this error-system may also be written as

$$
\dot{\xi}=Z_{1}(\xi) v_{1}+Z_{2} v_{2}+p_{\xi}\left(\xi, v_{r}\right)
$$

with $Z_{1}$, and $Z_{2}$ defined by (17), and

$$
p_{\xi}\left(\xi, v_{r}\right)=\left(\begin{array}{c}
p_{g}\left(\tilde{g}, v_{r}\right) \\
0_{2 \times 1}
\end{array}\right)
$$

A dynamic extension, corresponding to the lifting of $Z_{1}$ and $Z_{2}$ to $\bar{Z}_{1}$ and $\bar{Z}_{2}$ evoked in Theorem 1 is

$$
\dot{\bar{\xi}}=\bar{Z}_{1}(\bar{\xi}) v_{1}+\bar{Z}_{2} v_{2}+\bar{p}_{\bar{\xi}}\left(\bar{\xi}, v_{r}, \dot{\eta}_{r}\right)
$$

with $\bar{p}_{\bar{\xi}}=\left(p_{\xi}^{\prime}, 0\right)^{\prime}$. The feedback control design reported in the next two sections uses the left-invariance of $\bar{Z}_{1}$ and $\bar{Z}_{2}$, and the transverse functions determined in Section 4.3, in order to practically stabilize the point $\xi=0$ for any reference trajectory $g_{r}($.$) , and asymptotically stabilize this point in the particular case$ of feasible trajectories composed of straight lines and circles.

\subsection{Practical stabilizers}

We first recall how Theorem 1 can be used for control design purposes $[8$. Let $\bar{f}$ denote a smooth function defined on $K \times \mathbb{R}$, with $K$ a $\ell$-dimensional compact manifold. Let $\left(\frac{\partial \bar{f}}{\partial \alpha}\right)_{i}, i=1, \ldots, \ell$ denote a family of v.f. such that - for short, and with a slight abuse of notation

$$
\dot{\bar{f}}(\alpha, t)=\frac{\partial \bar{f}}{\partial \alpha}(\alpha, t) \omega+\frac{\partial \bar{f}}{\partial t}(\alpha, t)
$$

for some $\ell$-dimensional "frequency" vector $\omega$. Consider the "modified" error $\bar{z}=\bar{\xi} \bullet \bar{f}^{-1}$. Several indexes are purposely skipped in forthcoming relations in order to lighten up the notation. From (2) and (31), and using the invariance of $\bar{Z}_{1}$ and $\bar{Z}_{2}$,

$$
\begin{aligned}
\dot{\bar{z}} & =d R_{\bar{f}-1}(\bar{\xi})\left(\dot{\bar{\xi}}-d L_{\bar{z}}(\bar{f}) \dot{\bar{f}}\right) \\
& =d R_{\bar{f}-1}(\bar{\xi}) d L_{\bar{z}}(\bar{f})\left(\bar{Z}_{1}(\bar{f}) v_{1}+\bar{Z}_{2} v_{2}-\frac{\partial \bar{f}}{\partial \alpha} \omega\right)+d R_{\bar{f}-1}(\bar{\xi})\left(\bar{p}_{\bar{\xi}}-d L_{\bar{z}}(\bar{f}) \frac{\partial \bar{f}}{\partial t}\right)
\end{aligned}
$$

This relation may also be written as

$$
\dot{\bar{z}}=\bar{B}\left(\begin{array}{c}
v \\
\omega
\end{array}\right)+\bar{p}
$$


with

$$
\begin{aligned}
& \bar{p}=d R_{\bar{f}^{-1}}(\bar{\xi})\left(\bar{p}_{\bar{\xi}}-d L_{\bar{z}}(\bar{f}) \frac{\partial \bar{f}}{\partial t}\right) \\
& \bar{B}=d R_{\bar{f}^{-1}}(\bar{\xi}) d L_{\bar{z}}(\bar{f}) H(\alpha, t) \\
& H(\alpha, t)=\left(\begin{array}{lll}
\bar{Z}_{1}(\bar{f}(\alpha, t)) & \bar{Z}_{2} & -\frac{\partial \bar{f}}{\partial \alpha}(\alpha, t)
\end{array}\right)
\end{aligned}
$$

The transverslity of the function $\bar{f}$ w.r.t. $\bar{Z}_{1}$ and $\bar{Z}_{2}$ means that the $6 \times(\ell+2)$ dimensional matrix-valued function $H$ is of full rank (equal to 6) $\forall(\alpha, t)$. This in turn implies that $\bar{B}$ is a full-rank matrix-valued function. Using the pair $(v, \omega)$ as an extended control input, it is then simple to design a feedback control which exponential stabilizes $\bar{z}=0$. Note, however, that we are only interested here in controlling the first five components of $\bar{z}$, since $\bar{z}_{6}=\left(\bar{\xi}_{6}-f_{6}\right)$ involves the variable $\bar{\xi}_{6}$ which does not correspond to any physical entity. Let $z$ denote the vector regrouping these five components, $p$ denote the first five components of $\bar{p}$, and $B$ denote the $5 \times(\ell+2)$-dimensional matrix formed with the first five lines of $\bar{B}$, so that

$$
\dot{z}=B\left(\begin{array}{c}
v \\
\omega
\end{array}\right)+p
$$

One verifies that $z, \bar{B}$ (and thus $B$ ), as well as $\bar{p}$ (and thus $p$ ) do not depend on the "extra" variable $\bar{\xi}_{6}$, so that these terms can be calculated without having to compute this variable. Now, since $B$ is also of full rank (equal to 5), it is simple to define $v$ and $\omega$ so as to achieve the exponential stabilization of $z=0$. Take, for instance,

$$
\left(\begin{array}{c}
v \\
\omega
\end{array}\right)=-B^{\dagger}(k z+p), \quad k>0
$$

with $B^{\dagger}=B^{\prime}\left(B B^{\prime}\right)^{-1}$ the Moore-Penrose right pseudo-inverse of $B$. This yields the closed-loop equation $\dot{z}=-k z$ so that the tracking error $g_{r}^{-1} g$ converges to $\bar{f}_{g}$-the vector composed of the first three components of $\bar{f}$ - and $\eta$ converges to $\bar{f}_{\eta}$-the vector composed of the fourth and fifth components of $\bar{f}$. There remains to specify $\bar{f}$. A possibility is given by $\bar{f}=f^{\varepsilon}$, with $f^{\varepsilon}$ defined in Theorem 2 and constant parameters $\varepsilon, a_{1,2,3}, d_{1,2,3}$ that ensure the transversality of $f^{\epsilon}$. Then, $\alpha=\left(\theta_{1}, \theta_{2}, \theta_{3}, Q\right)$ and $\bar{f}$ ranges in a compact set, so that practical stabilization of any reference trajectory for the general two-trailers system follows. Decreasing $\varepsilon$ improves the tracking precision, since $f^{\varepsilon}$ uniformly tends to the null vector when $\varepsilon$ tends to zero, but also renders $\left(B B^{\prime}\right)$ closer to a singular matrix with the common consequence of increasing the intensities of the control inputs $v$ and $\omega$, and thus the frequency of maneuvers, especially when the reference trajectory is not feasible.

\subsection{Asymptotic stabilization of reference trajectories with constant curvature}

It is possible to modify the practical stabilizer (34) so as to allow for the asymptotic stabilization of feasible reference trajectories. Rewrite System (33) as

$$
\dot{z}=B\left(\begin{array}{c}
\tilde{v} \\
\omega
\end{array}\right)+\tilde{p}
$$

with $\tilde{v}=\left(v_{1}-v_{r, 1}, v_{2}-v_{r, 3}\right)^{\prime}$ and

$$
\tilde{p}=p+B\left(\begin{array}{c}
v_{r, 1} \\
v_{r, 3} \\
0_{3 \times 1}
\end{array}\right)
$$

Exponential stabilization of $z=0$ can be obtained by using (34) with $v$ and $p$ replaced by $\tilde{v}$ and $\tilde{p}$ respectively. A more general control expression, yielding the same closed-loop dynamics for $z$ (and thus also unconditional practical stabilization of any reference trajectory) is

$$
\left(\begin{array}{c}
\tilde{v} \\
\omega
\end{array}\right)=-B_{W}^{+}(k z+\tilde{p})-\left(I_{8}-B^{\dagger} B\right) \lambda
$$

$\mathrm{RR} \mathrm{n}^{\circ} 7938$ 
with

$$
B_{W}^{+}=W B^{\prime}\left(B W B^{\prime}\right)^{-1}
$$

a general right pseudo-inverse of $B, W$ any $(\ell+2) \times(\ell+2)$ symmetric positive definite (s.p.d.) matrix, and $\lambda$ any function of time whose importance in relation to the complementary objective of stabilizing feasible reference trajectories asymptotically is pointed out and explicited in [15]. More precisely, asymptotic stabilization of feasible reference trajectories relies on specific choices of $i$ ) the transverse function $\bar{f}$, ii) the "correction term" $\lambda$, and iii) the weight matrix $W$. Possible choices for $\bar{f}$ and $\lambda$ are detailed next.

In view of the definition of $z$, the convergence of $z$ to zero yields the convergence of $\tilde{g}$ and $\left(\eta-\eta_{r}\right)$ to zero when $\bar{f}_{g}$ converges to the unit element of $\mathbb{S E}(2)$ and $\bar{f}_{\eta}$ converges to $\left(\eta_{r, 1}, \eta_{r, 2}, 0\right)^{\prime}$. This suggests to use the function $\bar{f}$ defined by

$$
\bar{f}(\alpha, t)=f_{r}(t) \bullet\left(f^{\varepsilon}\left(\alpha^{*}\right)\right)^{-1} \bullet f^{\varepsilon}(\alpha)
$$

with

$$
\alpha=\left(\theta_{1}, \theta_{2}, \theta_{3}, Q\right), \quad f_{r}(t)=\left(0,0,0, \eta_{r, 1}(t), \eta_{r, 2}(t), 0\right)^{\prime}
$$

and $\alpha^{*}$ a suitable constant element of $\mathbb{T}^{3} \times \mathbb{S O}(3)$. Indeed, the transversality of this function is ensured by the transversality of $f^{\varepsilon}$ and $\bar{f}\left(\alpha^{*}, t\right)=f_{r}(t)=\left(0,0,0, \eta_{r, 1}(t), \eta_{r, 2}(t), 0\right)^{\prime}$. It thus suffices that $\alpha$ converges to $\alpha^{*}$ to obtain the desired convergence result. Following [15], we propose to achieve the convergence of $\alpha$ to $\alpha^{*}$ via the combined choices of $\alpha^{*}$ and the function $\lambda$ in (36). As already pointed out, the choice of this latter function does not affect the exponential convergence of $z$ to zero. On the other hand, it influences the system's zero-dynamics associated with $z=0$. We propose the function defined by

$$
\lambda=k_{\lambda}\left(0,0, \sin \left(\theta_{1}-\theta_{1}^{*}\right), \bar{k}_{\lambda} q_{s} q_{v}^{\prime}, \sin \left(\theta_{2}-\theta_{2}^{*}\right), \sin \left(\theta_{3}-\theta_{3}^{*}\right)\right)^{\prime}
$$

with $k_{\lambda}, \bar{k}_{\lambda}$ positive gains, and $\left(q_{s}, q_{v}\right)$ the scalar and vectorial part of the quaternion associated with $\left(Q^{*}\right)^{-1} Q$. The expression (39) is inspired by [15], where a similar function -used for a lower-order systemis shown to yield the convergence of $\alpha$ to a suitably chosen element $\alpha^{*}$ whose correspondance, for the system here considered, is

$$
\alpha^{*}=\left(\theta_{1}^{*}, \theta_{2}^{*}, \theta_{3}^{*}, Q^{*}\right)=\left(-\pi / 2,-\pi / 2,0, I_{3}\right)
$$

The proof of convergence remains to be worked out in the present case, but simulation results alike these reported in Section 5 tend to show that this property is granted for feasible reference trajectories (for which $v_{r, 2}=0$ ) with a constant curvature.

\subsection{Linear asymptotic stabilizers of reference trajectories with constant cur- vature}

Since the linearization of the two-trailers system is controllable along trajectories with a constant curvature, linear asymptotic stabilizers of such trajectories can be obtained by applying classical control design techniques. A possible solution of this type is derived next for comparison purposes. Let $\eta_{r}$ denote the reference shape vector defined by (20), and $\tilde{\eta}:=\eta-\eta_{r}$. Define the following variables:

$$
x_{i}=\tilde{g}_{i}(i=1,2,3), x_{4}=\frac{\tilde{\eta}_{1}-\tilde{\eta}_{2}}{2}+\tilde{g}_{3}, x_{5}=\frac{\tilde{\eta}_{1}+\tilde{\eta}_{2}}{2}+\tilde{g}_{2}
$$

Proposition 2. The feedback law

$$
\begin{aligned}
\tilde{v}_{1}= & -v_{r, 3} x_{2}-k_{4}\left|v_{r, 1}\right| x_{1} \\
\tilde{v}_{2}= & \frac{v_{r, 1}}{1+c_{r}^{2}}\left(k_{3}\left(x_{4}-\left(1+c_{r}^{2}\right) x_{3}\right) \operatorname{sign}\left(v_{r, 1}\right)+\cdots\right. \\
& \left.\left(1+k_{2}\right)\left(x_{5}-\left(1+c_{r}^{2}\right) x_{2}\right)+k_{1} x_{4} \operatorname{sign}\left(v_{r, 1}\right)+k_{0} x_{5}\right)
\end{aligned}
$$

with $k_{4}>0$ and $k_{0,1,2,3}$ any set of gain values that make the polynomial $s^{4}+k_{3} s^{3}+k_{2} s^{2}+k_{1} s+k_{0}$ Hurwitz-stable, make the reference trajectory $\left(g_{r}, \eta_{r}\right)$ localy exponentially stable provided that $\left.i\right) c_{r}$ is 
constant, ii) $v_{r, 1}$ is bounded, and iii) there exist constant $T, \delta>0$ such that

$$
\forall t \in \mathbb{R}_{+}, \quad \int_{t}^{t+T}\left|v_{r, 1}(s)\right| d s \geq \delta
$$

The proof is given in the appendix.

\section{Simulation results}

For the reported simulations, the time history of the reference frame velocity $v_{r}$ is summarized in the following table. Feasible trajectories correspond to the case when $v_{r, 2}=0, \forall t$. Controllability of the system's linear approximation along such trajectories further requires that $v_{r, 1} \neq 0$.

\begin{tabular}{|l|l|}
\hline$t \in(s)$ & $v_{r}=(\mathrm{m} / \mathrm{s}, \mathrm{m} / \mathrm{s}, \mathrm{rad} / \mathrm{s})^{\prime}$ \\
\hline$[0,5)$ & $(0,0,0)^{\prime}$ \\
{$[5,13)$} & $(0.6,0,0.05(t-5))^{\prime}$ \\
{$[13,21)$} & $(0.6,0,0.4-0.05(t-13))^{\prime}$ \\
{$[21,28)$} & $(0.8,0,0)^{\prime}$ \\
{$[28,35)$} & $(0,0,0.4)^{\prime}$ \\
{$[35,42)$} & $(0,-0.5,0)^{\prime}$ \\
{$[42,44)$} & $(-0.75(t-42), 0,0.3(t-42))^{\prime}$ \\
{$[44,50)$} & $(-1.5,0,0.6)^{\prime}$ \\
{$[50,52)$} & $(-1.5,0,0.6-0.6(t-50))^{\prime}$ \\
{$[52,57)$} & $(-1.5,0,-0.6)^{\prime}$ \\
{$[57,59)$} & $(-1.5,0,-0.6+0.3(t-57))^{\prime}$ \\
{$[59,60)$} & $(0,0,0)^{\prime}$ \\
\hline
\end{tabular}

\subsection{TF controller}

The control law is defined by (36) with

$$
W=\operatorname{Diag}(1,1,100,100,100,100,100,100), \quad k=3
$$

$\lambda$ and $\alpha^{*}$ given by (39) and (40) respectively, $k_{\lambda}=5\left|v_{r, 1}\right|$, and $\bar{k}_{\lambda}=20$. The transverse function used in the control law is given by (38), with $f^{\varepsilon}$ given by (27) and the parameters $\varepsilon, a_{1,2,3}, d_{1,2,3}$ of this function chosen as follows

$$
\begin{gathered}
\varepsilon=0.6, a_{1}=\left(0.4 \operatorname{sign}\left(v_{r, 1}\right), 1.1\right), a_{2}=\left(2.5 \operatorname{sign}\left(v_{r, 1}\right), 3\right) \\
a_{3}=\left(1.5,-6 \operatorname{sign}\left(v_{r, 1}\right)\right),\left(d_{1}, d_{2}, d_{3}\right)=-\operatorname{sign}\left(v_{r, 1}\right)(1.6,1,1)
\end{gathered}
$$

In order to avoid singularities of the reference shape vector $\eta_{r}$ at $v_{r, 1}=0$ (see (201)), and discontinuities at time-instants when the reference velocity is itself discontinuous, the function defined by (20) is approximated by the solution to the following differential equation

$$
\dot{\eta}_{r, i}=-k_{\eta}\left(\eta_{r, i}-\frac{v_{r, 3} v_{r, 1}}{v_{r, 1}^{2}+\delta^{2}}\right)
$$

Choosing $\delta$ small and $k_{\eta}$ large yields reference values close to these given by (20) when $v_{r, 1}$ is away from zero, and close to zero when $v_{r, 1}=0$. The simulation values are $\delta=0.01$ and $k_{\eta}=5$. Note that, by design of the TF control solution, the practical stabilization property -contrary to asymptotic stabilization- is basically independent of the choice of $\eta_{r}$ provided that this term is uniformly bounded. Another practical modification concerns the parameter $\varepsilon$ involved in the term $f^{\varepsilon}\left(\alpha^{*}\right)$ that we have replaced by

$$
\tilde{\varepsilon}=\varepsilon\left(\tanh \left(v_{r, 1} / \mu\right)\right)^{2}
$$

$\mathrm{RR} \mathrm{n}^{\circ} 7938$ 
with $\mu$ a small positive number, equal to 0.01 in the simulation. Then, $\tilde{\varepsilon} \approx \varepsilon$ when $v_{r, 1}$ is away from zero, and $\tilde{\varepsilon}=0$ when $v_{r, 1}=0$ so that the precompensation term $f^{\tilde{\varepsilon}}\left(\alpha^{*}\right)$ in (38) vanishes in this case. This is coherent with the fact that a non-zero precompensation is not useful when $v_{r, 1}=0$ because asymptotic stabilization of fixed points is not achievable with the present solution.

Fig. 2 shows the trajectories of the vehicule (full line) and of the reference frame (dashed line) in Cartesian space. The initial position coordinates of the mid-vehicle at $t=0$ are $(x, y)=(-2,1)$, whereas these of the reference frame are $\left(x_{r}, y_{r}\right)=(0,0)$. The figure also illustrates the control's capacity to maintain the vehicle's position nearby the origin of the reference frame, whatever the trajectory of this frame.

Fig. 3 shows the time evolution of the norm of $z$. Picks occur at time-instants when the transverse function coefficients $a_{i}$ vary abruptly due to sign changes of $v_{r, 1}$ and also fast variations of $\tilde{\varepsilon}$ when $v_{r, 1}$ gets close to zero. These variations could be compensated for at the control level so as to maintain $z$ equal to zero, but we have chosen not to do so for the sake of simplicity and to illustrate an aspect of the controller's robustness.

Finally, Fig. 4 shows the time evolution of Cartesian tracking errors, the boundedness of the orientation error, and the convergence of these errors to zero when the reference trajectory is feasible and the curvature $c_{r}$ is constant, i.e when $t \in[21 ; 28], t \in[44 ; 50]$ and $t \in[52 ; 57]$.

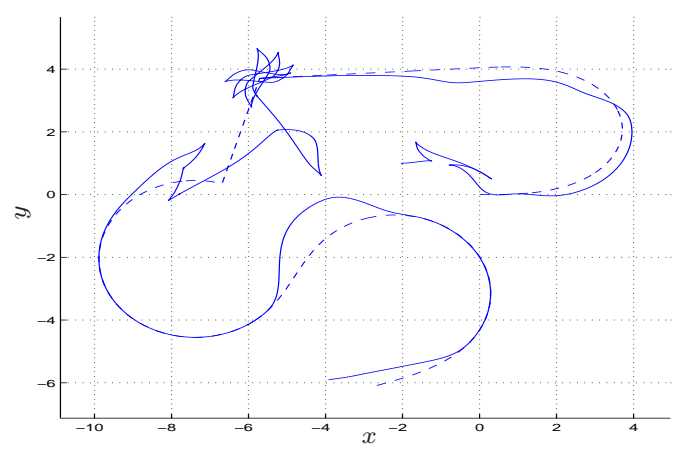

Figure 2: TF controller. Trajectories in the $x-y$ plane

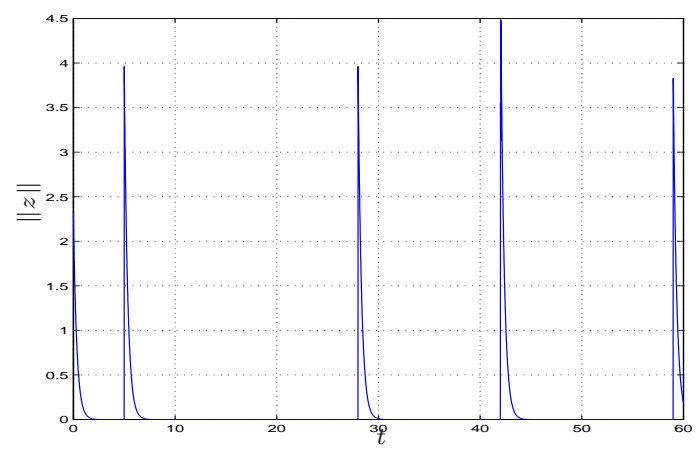

Figure 3: Norm of $z$ versus time 


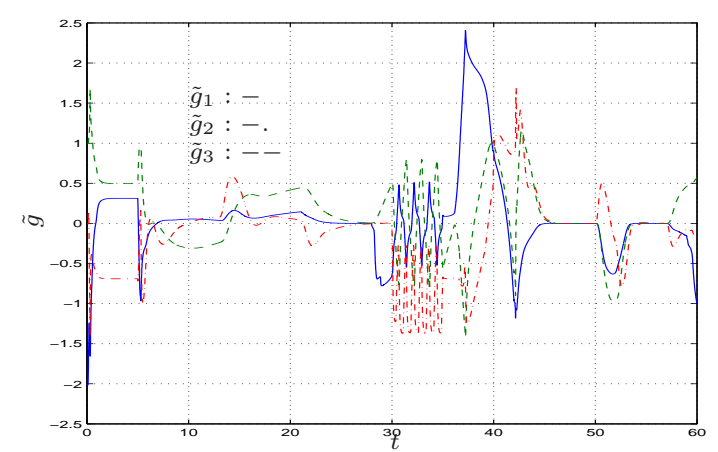

Figure 4: TF controller. Tracking errors in $\mathbb{S E}(2)$

\subsection{Linear controller}

For comparison purposes, tracking of the same reference frame trajectory with the linear controller of Section 4.7 has also been simulated. The control gains $k_{i}$ of the linear controller are defined by $k_{4}=2$ and

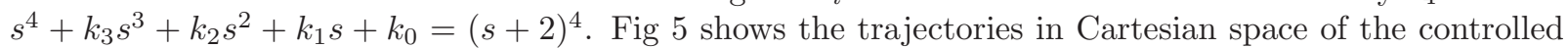
mid-vehicule (full line) and of the reference frame (dashed line), and Fig 6 shows the time evolution of the Cartesian tracking errors. When the reference trajectory is feasible (or almost feasible) and persistently exciting, the vehicule's behavior is similar to the one obtained with the TF controller. On the other hand, and as expected, the tracking errors can become arbitrarily large when the reference motion is not feasible, as in the case of pure lateral reference motion when $t \in[35 ; 42]$. Another shortcoming of this solution, not visible on these figures, is the occurence of the knife-edge effect when the reference motion is a pure rotation, when $t \in[28 ; 35]$. This effect, of no consequence in simulation, would of course be problematic in practice. A third shortcoming of the linear controller is its incapacity to reduce the Cartesian error when the reference frame is motionless, when $t \in[0 ; 5]$.

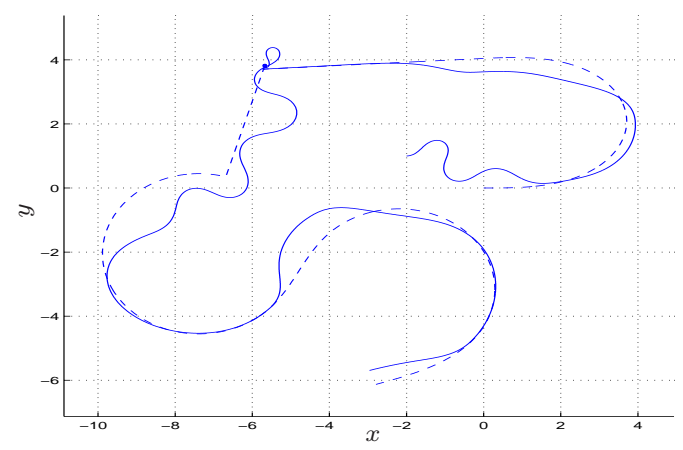

Figure 5: Linear controller. Trajectories in the $x-y$ plane

$\mathrm{RR} \mathrm{n}^{\circ} 7938$ 


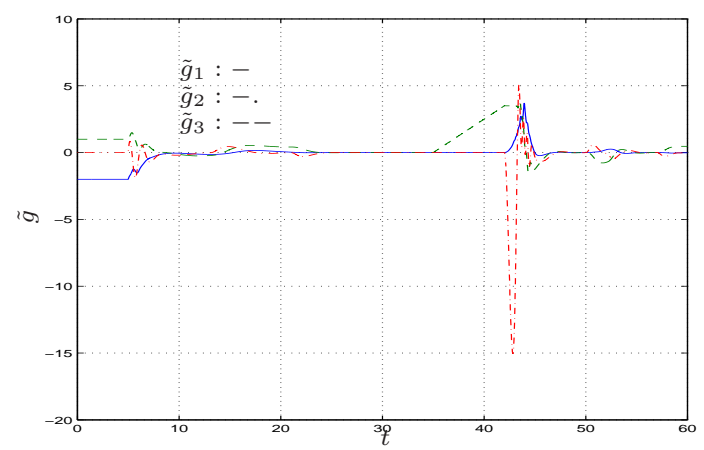

Figure 6: Linear controller. Tracking errors in $\mathbb{S E}(2)$

\section{Conclusion}

A new solution to the trajectory tracking problem has been proposed for the general two-trailers system. Unlike more classical, simpler, but also far less versatile control solutions proposed for this system, it yields practical stabilization of any reference trajectory, whether it is or is not feasible. A direct follow-up of the present work is a completion of the asymptotic stability analysis in the case of feasible trajectories with constant curvature. Other possible extensions include, e.g., the treatment of a larger number of trailers and experimental validation of the proposed solution.

\section{References}

[1] R. Murray and S. Sastry, "Steering nonholonomic systems in chained form," in IEEE Conf. on Decision and Control, 1991, pp. 1121-1126.

[2] O. J. Sørdalen, "Conversion of the kinematics of a car with $n$ trailers into a chained form," in $I E E E$ Conf. on Robotics and Automation, 1993, pp. 382-387.

[3] M. Fliess, J. Lévine, P. Martin, and P. Rouchon, "Flatness and defect of non-linear systems: introductory theory and examples," International Journal of Control, vol. 61, pp. 1327-1361, 1995.

[4] P. Rouchon, M. Fliess, J. Lévine, and P. Martin, "Flatness, motion planning and trailer systems," in IEEE Conf. on Decision and Control, 1993, pp. 2700-2705.

[5] D. Lizárraga, P. Morin, and C. Samson, "Chained form approximation of a driftless system. Application to the exponential stabilization of the general N-trailer system," International Journal of Control, vol. 74, pp. 1612-1629, 2001.

[6] M. Venditelli and G. Oriolo, "Stabilization of the general two-trailer system," in IEEE Conf. on Robotics and Automation, 2000, pp. 1817-1823.

[7] P. Morin and C. Samson, "A characterization of the Lie algebra rank condition by transverse periodic functions," SIAM Journal on Control and Optimization, vol. 40, no. 4, pp. 1227-1249, 2001.

[8] —_ "Practical stabilization of driftless systems on Lie groups: the transverse function approach," IEEE Trans. on Automatic Control, vol. 48, pp. 1496-1508, 2003.

[9] — - "Feedback control of a wheeled snake mechanism with the transverse function approach," in IEEE Conf. on Decision and Control, 2010, pp. 1991-1998. 
[10] M. Venditelli, J.-P. Laumond, and G. Oriolo, "Steering nonholonomic systems via nilpotent approximations: The general two-trailer system," in IEEE Conf. on Robotics and Automation, 1999, pp. 823-829.

[11] P. Morin and C. Samson, "Control of nonholonomic mobile robots based on the transverse function approach," IEEE Trans. on Robotics, vol. 25, pp. 1058-1073, 2009.

$[12] —$ - "Stabilization of trajectories for systems on Lie groups. Application to the rolling sphere," in IFAC World Congress, 2008, pp. 508-513.

[13] — - "Transverse function on special orthogonal groups for vector fields satisfying the LARC at the order one," in IEEE Conf. on Decision and Control, 2009, pp. 7472-7477.

[14] M. Ishikawa, P. Morin, and C. Samson, "Tracking control of the trident snake robot with the transverse function approach," in IEEE Conf. on Decision and Control, 2009, pp. 4137-4143.

[15] P. Morin and C. Samson, "Control of two-steering-wheels vehicles with the transverse function approach," in IEEE Conf. on Decision and Control, 2011, pp. 7349-7355.

[16] S. Helgason, Differential geometry, Lie groups, and symmetric spaces. Academic Press, 1978.

[17] H. Khalil, Nonlinear systems, 3rd ed. Prentice Hall, 2002.

\section{Appendix 1: Proof of Theorem 2}

The following notation is used in the proof.

- We denote by $\ell(k)$ the "length" of the Lie bracket associated with $X_{k}$, i.e. $\ell(1)=\ell(2)=1, \ell(3)=2$, $\ell(4)=\ell(5)=3, \ell(6)=\ell(7)=4$.

- We denote by $O(\varepsilon)$ any function which depends continuously on a parameter $\varepsilon$ and such that $O(0)$ is the null function.

- For any vector $u \in \mathbb{R}^{n}, u_{p \rightarrow q}$ denotes the sub-vector of $u$ equal to $\left(u_{p}, u_{p+1}, \cdots, u_{q}\right)^{T}$.

- For any v.f. $Z, Y \in \mathfrak{g}$ and any $k \in \mathbb{N}$, $\operatorname{ad}^{k}(Z)(Y)$ is the v.f. defined recursively by $\operatorname{ad}^{0}(Z)(Y)=Y$ and $\operatorname{ad}^{k}(Z)(Y)=\left[Z, \operatorname{ad}^{k-1}(Z)(Y)\right]$ for $k \geq 1$.

We show below that the function $f^{\varepsilon}$ defined by (27) is a TF for $\varepsilon$ small enough and for an adequate choice of the parameters $a_{1}, a_{2}, a_{3}$, and $D$.

From (27), let us rewrite $f^{\varepsilon}$ as

$$
f^{\varepsilon}=f_{3}^{\varepsilon} f_{2}^{\varepsilon} f_{1}^{\varepsilon}
$$

where the $\bullet$ symbol for the group operation is omitted to simplify the notation, and

$$
f_{1}^{\varepsilon}=\exp \left(X \Delta_{\varepsilon} \mu_{a_{1}}\right), f_{2}^{\varepsilon}=\exp \left(X \Delta_{\varepsilon} \nu_{D}\right), f_{3}^{\varepsilon}=\exp \left(X \Delta_{\varepsilon} \delta_{a_{2}, a_{3}}\right)
$$

with $X=\left[X_{1}, \ldots, X_{7}\right]$,

$$
\mu_{a_{1}}\left(\theta_{1}\right)=\left(\begin{array}{c}
a_{1,1} \sin \theta_{1} \\
a_{1,2} \cos \theta_{1} \\
0 \\
\frac{a_{1,1}^{2} a_{1,2}}{3} \cos \theta_{1} \\
-\frac{a_{1,1} a_{1,2}^{2}}{3} \sin \theta_{1} \\
0_{2 \times 1}
\end{array}\right), \delta_{a_{2}, a_{3}}\left(\theta_{2}, \theta_{3}\right)=\left(\begin{array}{c}
a_{2,1} \sin \theta_{2} \\
a_{3,1} \sin \theta_{3} \\
0 \\
a_{2,2} \cos \theta_{2} \\
a_{3,2} \cos \theta_{3} \\
0_{2 \times 1}
\end{array}\right), \nu_{D}(Q)=\left(\begin{array}{c}
D Q e_{1} \\
P \bar{D} Q e_{3} \\
0_{2 \times 1}
\end{array}\right)
$$

and

$$
\begin{aligned}
\Delta_{\varepsilon} & =\operatorname{Diag}\left(\varepsilon, \varepsilon, \varepsilon^{2}, \varepsilon^{3}, \varepsilon^{3}, \varepsilon^{4}, \varepsilon^{4}\right) \\
& =\operatorname{Diag}\left(\varepsilon^{\ell(1)}, \varepsilon^{\ell(2)}, \varepsilon^{\ell(3)}, \varepsilon^{\ell(4)}, \varepsilon^{\ell(5)}, \varepsilon^{\ell(6)}, \varepsilon^{\ell(7)}\right) \quad, \quad P=\left(\begin{array}{ccc}
0 & -1 & 0 \\
1 & 0 & 0
\end{array}\right)
\end{aligned}
$$

$\mathrm{RR} \mathrm{n}^{\circ} 7938$ 
Note the slight abuse of notation since $\mu_{a_{1}}, \delta_{a_{2}, a_{3}}, \nu_{D}$, and $\Delta_{\varepsilon}$ so defined are not exactly the same as those defined in Section 4.3 (they have been extended as $\mathbb{R}^{7}$-valued functions). The resulting expression of $f^{\varepsilon}$, however, is the same.

\section{Expression of $\dot{f}^{\varepsilon}$}

We first establish the expression of $\dot{f}^{\varepsilon}$. The calculation makes use of the following classical formula 16 , Pg. 105] for the derivative of the exponential mapping on Lie groups:

$$
\frac{d}{d t} \exp (Z+t Y)_{\mid t=0}=\sum_{k=0}^{\infty} \frac{(-1)^{k}}{(k+1) !} \operatorname{ad}^{k}(Z)(Y)(\exp (Z))
$$

When $Z(t)$ denotes a time-varying vector field, the above expression yields

$$
\frac{d}{d t} \exp (Z(t))=\sum_{k=0}^{\infty} \frac{(-1)^{k}}{(k+1) !} \operatorname{ad}^{k}(Z(t))(\dot{Z}(t))(\exp (Z(t)))
$$

with $\dot{Z}(t)$ the time-varying vector field $\frac{d}{d t} Z(t)$. Expansion of the above series yields

$$
\frac{d}{d t} \exp (Z(t))=\left(\dot{Z}(t)-\frac{1}{2}[Z, \dot{Z}](t)+\frac{1}{6}[Z,[Z, \dot{Z}]](t)-\frac{1}{24}[Z,[Z,[Z, \dot{Z}]]](t)+\cdots\right)(\exp (Z(t)))
$$

The following lemma, whose proof is given further, is used for the calculation of $\dot{f}^{\varepsilon}$.

Lemma 1. For any $\varepsilon$ and any vectors $v, w \in \mathbb{R}^{7}$,

$$
\begin{gathered}
\operatorname{ad}^{1}\left(X \Delta_{\varepsilon} v\right)\left(X \Delta_{\varepsilon} w\right)=\left[X \Delta_{\varepsilon} v, X \Delta_{\varepsilon} w\right]=X \Delta_{\varepsilon}\left[\left(\begin{array}{c}
0_{2 \times 1} \\
M\left(v_{1 \rightarrow 3} \times w_{1 \rightarrow 3}\right) \\
v_{1} w_{4}-v_{4} w_{1} \\
v_{2} w_{5}-v_{5} w_{2}
\end{array}\right)+O(\varepsilon)\right] \\
\operatorname{ad}^{2}\left(X \Delta_{\varepsilon} v\right)\left(X \Delta_{\varepsilon} w\right)=X \Delta_{\varepsilon}\left[\left(\begin{array}{c}
0_{3 \times 1} \\
v_{1 \rightarrow 2}\left(v_{1} w_{2}-v_{2} w_{1}\right) \\
v_{1}\left(v_{1} w_{3}-v_{3} w_{1}\right) \\
v_{2}\left(v_{2} w_{3}-v_{3} w_{2}\right)
\end{array}\right)+O(\varepsilon)\right] \\
\operatorname{ad}^{3}\left(X \Delta_{\varepsilon} v\right)\left(X \Delta_{\varepsilon} w\right)=X \Delta_{\varepsilon}\left[\left(\begin{array}{c}
0_{5 \times 1} \\
v_{1}^{2}\left(v_{1} w_{2}-v_{2} w_{1}\right) \\
v_{2}^{2}\left(v_{1} w_{2}-v_{2} w_{1}\right)
\end{array}\right)+O(\varepsilon)\right]
\end{gathered}
$$

and

$$
\operatorname{ad}^{k}\left(X \Delta_{\varepsilon} v\right)\left(X \Delta_{\varepsilon} w\right)=X \Delta_{\varepsilon} O(\varepsilon), \quad \forall k \geq 4
$$

with

$$
M=\left(\begin{array}{c}
e_{3}^{T} \\
P
\end{array}\right)=\left(\begin{array}{ccc}
0 & 0 & 1 \\
0 & -1 & 0 \\
1 & 0 & 0
\end{array}\right)
$$

\section{Calculation of $\dot{f}_{1}^{\varepsilon}$ :}

From (45), $f_{1}^{\varepsilon}=\exp (Z)$ with $Z=X \Delta_{\varepsilon} \mu_{a_{1}}$. Using Lemma 1 1 above and the expression (46) of $\mu_{a_{1}}$, the 
following expressions are obtained:

$$
\begin{aligned}
& \dot{Z}=X \Delta_{\varepsilon} \dot{\mu}_{a_{1}} \\
& =X \Delta_{\varepsilon}\left(a_{1,1} \cos \theta_{1},-a_{1,2} \sin \theta_{1}, 0,-\frac{a_{1,1}^{2} a_{1,2}}{3} \sin \theta_{1},-\frac{a_{1,1} a_{1,2}^{2}}{3} \cos \theta_{1}, 0,0\right)^{T} \dot{\theta}_{1} \\
& {[Z, \dot{Z}]=\operatorname{ad}^{1}(Z)(\dot{Z})} \\
& =X \Delta_{\varepsilon}\left[\left(0,0,-a_{1,1} a_{1,2}, 0,0, c_{a_{1}}\left(\theta_{1}\right), c_{a_{1}}\left(\theta_{1}\right)\right)^{T}+O(\varepsilon)\right] \dot{\theta}_{1} \\
& {[Z,[Z, \dot{Z}]]=\operatorname{ad}^{2}(Z)(\dot{Z})} \\
& \quad=X \Delta_{\varepsilon}\left[\left(0,0,0,-a_{1,1}^{2} a_{1,2} \sin \theta_{1},-a_{1,1} a_{1,2}^{2} \cos \theta_{1}, 0,0\right)^{T}+O(\varepsilon)\right] \dot{\theta}_{1} \\
& {[Z,[Z,[Z, \dot{Z}]]]=\operatorname{ad}^{3}(Z)(\dot{Z})} \\
& \quad=X \Delta_{\varepsilon}\left[\left(0,0,0,0,0, c_{a_{1}}\left(\theta_{1}\right), c_{a_{1}}\left(\theta_{1}\right)\right)^{T}+O(\varepsilon)\right] \dot{\theta}_{1} \\
& \operatorname{ad}^{k}(Z)(\dot{Z})=X \Delta_{\varepsilon} O(\varepsilon), \quad \forall k \geq 4
\end{aligned}
$$

where $c_{a}()$ is a generic notation for any smooth function that depends on a parameter $a$. Even though the different functions $c_{a_{1}}$ are not necessarily the same, the same notation is used because only the existence of these functions is needed here. Gathering all the above expressions of $\operatorname{ad}^{k}(Z)(\dot{Z})$ in (48) yields

$$
\dot{f}_{1}^{\varepsilon}=X\left(f_{1}^{\varepsilon}\right) \Delta_{\varepsilon}\left[\bar{\mu}_{a_{1}}\left(\theta_{1}\right)+O(\varepsilon)\right] \dot{\theta}_{1}
$$

with

$$
\bar{\mu}_{a_{1}}\left(\theta_{1}\right)=\left(a_{1,1} \cos \theta_{1},-a_{1,2} \sin \theta_{1}, \frac{a_{1,1} a_{1,2}}{2},-\frac{a_{1,1}^{2} a_{1,2}}{2} \sin \theta_{1},-\frac{a_{1,1} a_{1,2}^{2}}{2} \cos \theta_{1}, c_{a_{1}}\left(\theta_{1}\right), c_{a_{1}}\left(\theta_{1}\right)\right)^{T}
$$

\section{Calculation of $\dot{f}_{2}^{\varepsilon}$ :}

From (45), $f_{2}^{\varepsilon}=\exp (Z)$ with $Z=X \Delta_{\varepsilon} \nu_{D}$. From (46),

$$
\dot{Z}=X \Delta_{\varepsilon}\left(\begin{array}{c}
-D Q S\left(e_{1}\right) \\
-P \bar{D} Q S\left(e_{3}\right) \\
0_{2 \times 3}
\end{array}\right) \omega_{Q}
$$

with $S($.$) the 3 \times 3$ matrix-valued operator associated with the cross product on $\mathbb{R}^{3}$ and $\omega_{Q}$ the angular velocity vector associated with $Q$, i.e. $\dot{Q}=Q S\left(\omega_{Q}\right)$. It follows from the definition (23) of $D$ and $\bar{D}$ that, for any $Q \in \mathbb{S O}(3)$,

$$
\left(D Q e_{1}\right) \times\left(D Q S\left(e_{1}\right) \omega_{Q}\right)=\bar{D}\left(\left(Q e_{1}\right) \times\left(Q S\left(e_{1}\right) \omega_{Q}\right)\right)=\bar{D} Q S\left(e_{1}\right)^{2} \omega_{Q}
$$

Combining with (49) and (55) yields

$$
[Z, \dot{Z}]=X \Delta_{\varepsilon}\left(\begin{array}{c}
O_{2 \times 3}(\varepsilon) \\
-M \bar{D} Q S\left(e_{1}\right)^{2}+O(\varepsilon) \\
c_{D}(Q)+O(\varepsilon) \\
c_{D}(Q)+O(\varepsilon)
\end{array}\right) \omega_{Q}=X \Delta_{\varepsilon}\left(\begin{array}{c}
O_{2 \times 3}(\varepsilon) \\
-e_{3}^{T} \bar{D} Q S\left(e_{1}\right)^{2}+O(\varepsilon) \\
-P \bar{D} Q S\left(e_{1}\right)^{2}+O(\varepsilon) \\
c_{D}(Q)+O(\varepsilon) \\
c_{D}(Q)+O(\varepsilon)
\end{array}\right) \omega_{Q}
$$

It then follows from (50) that

$\mathrm{RR}^{\circ} 7938$

$$
[Z,[Z, \dot{Z}]]=X \Delta_{\varepsilon}\left(\begin{array}{c}
O_{3 \times 3}(\varepsilon) \\
-e_{1,2}^{T} D Q e_{1} e_{3}^{T} \bar{D} Q S\left(e_{1}\right)^{2}+O(\varepsilon) \\
c_{D}(Q)+O(\varepsilon) \\
c_{D}(Q)+O(\varepsilon)
\end{array}\right) \omega_{Q}
$$


with

$$
e_{1,2}^{T}=\left(\begin{array}{ccc}
1 & 0 & 0 \\
0 & 1 & 0
\end{array}\right)
$$

and from (51) that

$$
[Z,[Z,[Z, \dot{Z}]]]=X \Delta_{\varepsilon}\left(\begin{array}{c}
O_{5 \times 3}(\varepsilon) \\
c_{D}(Q)+O(\varepsilon) \\
c_{D}(Q)+O(\varepsilon)
\end{array}\right) \omega_{Q}
$$

Gathering all previous expressions of $\operatorname{ad}^{k} Z(\dot{Z})$ in (48) and using (52) yields

$$
\dot{f}_{2}^{\varepsilon}=X\left(f_{3}^{\varepsilon}\right) \Delta_{\varepsilon}\left[\bar{\nu}_{D}(Q)+O(\varepsilon)\right] \omega_{Q}
$$

with

$$
\bar{\nu}_{D}(Q)=\left(\begin{array}{c}
-e_{1,2}^{T} D Q S\left(e_{1}\right) \\
-e_{3}^{T} D Q S\left(e_{1}\right)+\frac{1}{2} e_{3}^{T} \bar{D} Q S\left(e_{1}\right)^{2} \\
\frac{1}{2} P \bar{D} Q\left(S\left(e_{1}\right)^{2}-2 S\left(e_{3}\right)\right)-\frac{1}{6} e_{1,2}^{T} D Q e_{1} e_{3}^{T} \bar{D} Q S\left(e_{1}\right)^{2} \\
c_{D}(Q) \\
c_{D}(Q)
\end{array}\right)
$$

Calculation of $\dot{f}_{3}^{\varepsilon}$ :

From (45), $f_{3}^{\varepsilon}=\exp (Z)$ with $Z=X \Delta_{\varepsilon} \delta_{a_{2}, a_{3}}$ so that,

$$
\begin{aligned}
\dot{Z}= & X \Delta_{\varepsilon}\left(a_{2,1} \cos \theta_{2}, 0,0,-a_{2,2} \sin \theta_{2}, 0,0,0\right)^{T} \dot{\theta}_{2} \\
& +X \Delta_{\varepsilon}\left(0, a_{3,1} \cos \theta_{3}, 0,0,-a_{3,2} \sin \theta_{3}, 0,0\right)^{T} \dot{\theta}_{3}
\end{aligned}
$$

Using Lemma 1, the following expressions are obtained:

$$
\begin{gathered}
{[Z, \dot{Z}]=\begin{array}{c}
X \Delta_{\varepsilon}\left(0,0,-a_{2,1} a_{3,1} \sin \theta_{3} \cos \theta_{2}, 0,0,-a_{2,1} a_{2,2}, 0\right)^{T} \dot{\theta}_{2} \\
+ \\
+X \Delta_{\varepsilon}\left(0,0, a_{2,1} a_{3,1} \sin \theta_{2} \cos \theta_{3}, 0,0,0,-a_{3,1} a_{3,2}\right)^{T} \dot{\theta}_{3}
\end{array}} \\
{[Z,[Z, \dot{Z}]]=\quad X \Delta_{\varepsilon}\left[\left(0,0,0,-a_{2,1}^{2} a_{3,1} \sin \theta_{3} \sin \theta_{2} \cos \theta_{2},-a_{2,1} a_{3,1}^{2} \sin ^{2} \theta_{3} \cos \theta_{2}, 0,0\right)^{T}+O(\varepsilon)\right] \dot{\theta}_{2}} \\
+X \Delta_{\varepsilon}\left[\left(0,0,0, a_{2,1}^{2} a_{3,1} \sin ^{2} \theta_{2} \cos \theta_{3}, a_{2,1} a_{3,1}^{2} \sin \theta_{2} \sin \theta_{3} \cos \theta_{3}, 0,0\right)^{T}+O(\varepsilon)\right] \dot{\theta}_{3}
\end{gathered}
$$

and

$$
\begin{aligned}
{[Z,[Z,[Z, \dot{Z}]]]=} & X \Delta_{\varepsilon}\left[\left(0,0,0,0,0,-a_{2,1}^{3} a_{3,1} \sin \theta_{3} \sin ^{2} \theta_{2} \cos \theta_{2},-a_{2,1} a_{3,1}^{3} \sin ^{3} \theta_{3} \cos \theta_{2}\right)^{T}+O(\varepsilon)\right] \dot{\theta}_{2} \\
& +X \Delta_{\varepsilon}\left[\left(0,0,0,0,0, a_{2,1}^{3} a_{3,1} \sin ^{3} \theta_{2} \cos \theta_{3}, a_{2,1} a_{3,1}^{3} \sin \theta_{2} \sin ^{2} \theta_{3} \cos \theta_{3}\right)^{T}+O(\varepsilon)\right] \dot{\theta}_{3}
\end{aligned}
$$

Gathering all these terms in (48) and using (52) again yields

$$
\dot{f}_{3}^{\varepsilon}=X\left(f_{3}^{\varepsilon}\right) \Delta_{\varepsilon}\left[\bar{\delta}_{a_{2}, a_{3}}\left(\theta_{2}, \theta_{3}\right)+O(\varepsilon)\right]\left(\dot{\theta}_{2}, \dot{\theta}_{3}\right)^{T}
$$

with

$$
\bar{\delta}_{a_{2}, a_{3}}\left(\theta_{2}, \theta_{3}\right)=\left(\begin{array}{cc}
a_{2,1} \cos \theta_{2} & 0 \\
0 & a_{3,1} \cos \theta_{3} \\
\frac{1}{2} a_{2,1} a_{3,1} \sin \theta_{3} \cos \theta_{2} & -\frac{1}{2} a_{2,1} a_{3,1} \sin \theta_{2} \cos \theta_{3} \\
-a_{2,2} \sin \theta_{2}-\frac{1}{6} a_{2,1}^{2} a_{3,1} \sin \theta_{3} \sin \theta_{2} \cos \theta_{2} & \frac{1}{6} a_{2,1}^{2} a_{3,1} \sin ^{2} \theta_{2} \cos \theta_{3} \\
-\frac{1}{6} a_{2,1} a_{3,1}^{2} \sin ^{2} \theta_{3} \cos \theta_{2} & -a_{3,2} \sin \theta_{3}+\frac{1}{6} a_{2,1} a_{3,1}^{2} \sin \theta_{2} \sin \theta_{3} \cos \theta_{3} \\
\frac{1}{2} a_{2,1} a_{2,2}+\frac{1}{24} a_{2,1}^{3} a_{3,1} \sin \theta_{3} \sin ^{2} \theta_{2} \cos \theta_{2} & -\frac{1}{24} a_{2,1}^{3} a_{3,1} \sin ^{3} \theta_{2} \cos \theta_{3} \\
\frac{1}{24} a_{2,1} a_{3,1}^{3} \sin ^{3} \theta_{3} \cos \theta_{2} & \frac{1}{2} a_{3,1} a_{3,2}-\frac{1}{24} a_{2,1} a_{3,1}^{3} \sin \theta_{2} \sin ^{2} \theta_{3} \cos \theta_{3}
\end{array}\right)
$$




\section{Expression of $\dot{f}^{\varepsilon}$ :}

For any time functions $g_{1}, g_{2}$ on $G$ such that $\dot{g}_{i}=X\left(g_{i}\right) v_{i}$ for $i=1,2$, the left-invariance of the vector fields $X_{i}$ implies that

$$
\frac{d}{d t}\left(g_{2} g_{1}\right)=d L_{g_{2} g_{1}}(e)\left[X(e) v_{1}+\operatorname{Ad}\left(g_{1}^{-1}\right) X(e) v_{2}\right]
$$

Recall that $L$ is the left-translation operator on $G$. By applying this relation to the product $f_{2}^{\varepsilon} f_{1}^{\varepsilon}$, it follows from (53) and (56) that

$$
\frac{d}{d t}\left(f_{2}^{\varepsilon} f_{1}^{\varepsilon}\right)=d L_{f_{2}^{\varepsilon} f_{1}^{\varepsilon}}(e)\left[X(e) \Delta_{\varepsilon}\left(\bar{\mu}_{a_{1}}+O(\varepsilon)\right) \dot{\theta}_{1}+\operatorname{Ad}\left(\left(f_{1}^{\varepsilon}\right)^{-1}\right) X(e) \Delta_{\varepsilon}\left(\bar{\nu}_{D}+O(\varepsilon)\right) \omega_{Q}\right]
$$

where the arguments of $\bar{\mu}_{a_{1}}$ and $\bar{\nu}_{D}$ have been omitted. Since $f^{\varepsilon}=f_{3}^{\varepsilon} f_{2}^{\varepsilon} f_{1}^{\varepsilon}$, we deduce from the above relation and (58) that

$$
\dot{f}^{\varepsilon}=d L_{f^{\varepsilon}}(e) \bar{H}_{a, D}^{\varepsilon}\left(\theta_{1}, \theta_{2}, \theta_{3}, Q\right)\left(\begin{array}{c}
\dot{\theta}_{1} \\
\omega_{Q} \\
\dot{\theta}_{2} \\
\dot{\theta}_{3}
\end{array}\right)
$$

with

$$
\bar{H}_{a, D}^{\varepsilon}=\left(X(e) \Delta_{\varepsilon}\left(\bar{\mu}_{a_{1}}+O(\varepsilon)\right) \quad \operatorname{Ad}\left(\left(f_{1}^{\varepsilon}\right)^{-1}\right) X(e) \Delta_{\varepsilon}\left(\bar{\nu}_{D}+O(\varepsilon)\right) \quad \operatorname{Ad}\left(\left(f_{2}^{\varepsilon} f_{1}^{\varepsilon}\right)^{-1}\right) X(e) \Delta_{\varepsilon}\left(\bar{\delta}_{a_{2}, a_{3}}+O(\varepsilon)\right)\right)
$$

\section{Sufficient conditions for the transversality of $f^{\varepsilon}$ :}

From the definition of a transverse function, (24), and the left invariance of the v.f. $X_{i}, f^{\varepsilon}$ is a TF for given values of $a_{1}, a_{2}, a_{3}, D$, and $\varepsilon>0$, if and only if,

$$
\forall \theta_{1}, \theta_{2}, \theta_{3}, Q, \quad \operatorname{Im}\left(\bar{H}_{a, D}^{\varepsilon}\left(\theta_{1}, \theta_{2}, \theta_{3}, Q\right)\right)+\operatorname{span}\left(X_{1}(e), X_{2}(e)\right)=\mathfrak{g}
$$

The proof of the existence of parameters $a_{1}, a_{2}, a_{3}, D$, and $\bar{\varepsilon}>0$ such that, for $\varepsilon \in(0, \bar{\varepsilon})$, (63) is satisfied relies on the following lemma (whose proof is given further).

Lemma 2. For any vectors $v, w \in \mathbb{R}^{7}$,

$$
\operatorname{Ad}\left(\exp \left(X \Delta_{\varepsilon} v\right)\right) X(e) \Delta_{\varepsilon} w=X(e) \Delta_{\varepsilon}(w+\gamma(v, w)+O(\varepsilon))
$$

with $\gamma$ a polynomial function of $v$ and $w$ which vanishes when $v=0$ or $w=0$. Furthermore,

1. $\gamma_{i}$ is a sum of monomials of the form $c_{i} v_{i_{1}} \cdots v_{i_{p}} w_{i_{p+1}}$ with $p \geq 1$ and $c_{i}$ some constant, and $\sum_{k=1}^{p+1} \ell\left(i_{k}\right)=\ell(i)$.

2. When $v_{3}=0$,

$$
\begin{aligned}
& \gamma_{3}(v, w)=v_{1} w_{2}-v_{2} w_{1} \\
& \gamma_{4}(v, w)=v_{1}\left(w_{3}+\frac{1}{2} \gamma_{3}(v, w)\right) \\
& \gamma_{5}(v, w)=v_{2}\left(w_{3}+\frac{1}{2} \gamma_{3}(v, w)\right)
\end{aligned}
$$

Since $f_{1}^{\varepsilon}=\exp \left(X \Delta_{\varepsilon} \mu_{a_{1}}\right)$, so that $\left(f_{1}^{\varepsilon}\right)^{-1}=\exp \left(-X \Delta_{\varepsilon} \mu_{a_{1}}\right)$, it follows from this lemma that

$$
\begin{aligned}
\operatorname{Ad}\left(\left(f_{1}^{\varepsilon}\right)^{-1}\right) X(e) \Delta_{\varepsilon}\left(\bar{\nu}_{D}+O(\varepsilon)\right) & =X(e) \Delta_{\varepsilon}\left(\bar{\nu}_{D}+O(\varepsilon)+\gamma\left(-\mu_{a_{1}}, \bar{\nu}_{D}+O(\varepsilon)\right)+O(\varepsilon)\right) \\
& =X(e) \Delta_{\varepsilon}\left(\bar{\nu}_{D}+\gamma\left(-\mu_{a_{1}}, \bar{\nu}_{D}\right)+O(\varepsilon)\right)
\end{aligned}
$$

$\mathrm{RR} \mathrm{n}^{\circ} 7938$ 
where the second equality comes from the fact that $\gamma$ is a polynomial function of its arguments. Similarly, using the fact that $f_{2}^{\varepsilon}=\exp \left(X \Delta_{\varepsilon} \nu_{D}\right)$ and the property $\operatorname{Ad}\left(g_{1} g_{2}\right)=\operatorname{Ad}\left(g_{1}\right) \operatorname{Ad}\left(g_{2}\right)$,

$$
\begin{array}{r}
\operatorname{Ad}\left(\left(f_{2}^{\varepsilon} f_{1}^{\varepsilon}\right)^{-1}\right) X(e) \Delta_{\varepsilon}\left(\bar{\delta}_{a_{2}, a_{3}}+O(\varepsilon)\right)=\operatorname{Ad}\left(\left(f_{2}^{\varepsilon}\right)^{-1}\right) \operatorname{Ad}\left(\left(f_{1}^{\varepsilon}\right)^{-1}\right) X(e) \Delta_{\varepsilon}\left(\bar{\delta}_{a_{2}, a_{3}}+O(\varepsilon)\right) \\
=\operatorname{Ad}\left(\left(f_{2}^{\varepsilon}\right)^{-1}\right) X(e) \Delta_{\varepsilon}\left(\bar{\delta}_{a_{2}, a_{3}}+\gamma\left(-\mu_{a_{1}}, \bar{\delta}_{a_{2}, a_{3}}\right)+O(\varepsilon)\right) \\
=X(e) \Delta_{\varepsilon}\left(\bar{\delta}_{a_{2}, a_{3}}+\gamma\left(-\mu_{a_{1}}, \bar{\delta}_{a_{2}, a_{3}}\right)+\gamma\left(-\nu_{D}, \bar{\delta}_{a_{2}, a_{3}}+\gamma\left(-\mu_{a_{1}}, \bar{\delta}_{a_{2}, a_{3}}\right)\right)+O(\varepsilon)\right)
\end{array}
$$

Therefore, using (62),

$$
\bar{H}_{a, D}^{\varepsilon}=X(e) \Delta_{\varepsilon}\left(M_{a, D}+O(\varepsilon)\right)
$$

with

$$
M_{a, D}=\left(\bar{\mu}_{a_{1}} \quad \bar{\nu}_{D}+\gamma\left(-\mu_{a_{1}}, \bar{\nu}_{D}\right) \quad \bar{\delta}_{a_{2}, a_{3}}+\gamma\left(-\mu_{a_{1}}, \bar{\delta}_{a_{2}, a_{3}}\right)+\gamma\left(-\nu_{D}, \bar{\delta}_{a_{2}, a_{3}}+\gamma\left(-\mu_{a_{1}}, \bar{\delta}_{a_{2}, a_{3}}\right)\right)\right)
$$

Since $M_{a, D}$ does not depend on $\varepsilon$, it follows from (66) that (63) is satisfied, for some $a_{1}, a_{2}, a_{3}, D$ and for $\varepsilon$ small provided that the $5 \times 6$ matrix

$$
H_{0}\left(\theta_{1}, \theta_{2}, \theta_{3}, Q\right)=\left(0_{5 \times 2} \quad I_{5,5}\right) M_{a, D}\left(\theta_{1}, \theta_{2}, \theta_{3}, Q\right)
$$

is of full rank for any $\theta_{1}, \theta_{2}, \theta_{3}, Q$. Note that this condition is only sufficient for the transversality of $f^{\varepsilon}$. In particular, when $\operatorname{dim}(G)<7$ the transversality of $f^{\varepsilon}$ can be achieved with a smaller rank of $H_{0}\left(\theta_{1}, \theta_{2}, \theta_{3}, Q\right)$.

\section{Full rankedness of $H_{0}$ :}

Let us decompose $H_{0}$ as follows:

$$
H_{0}=\left(\begin{array}{cc}
T & V \\
U & W
\end{array}\right)
$$

with

$$
\begin{aligned}
& T=\left(\begin{array}{ll}
\bar{\mu}_{a_{1}, 3 \rightarrow 5} & \bar{\nu}_{D, 3 \rightarrow 5}+\gamma_{3 \rightarrow 5}\left(-\mu_{a_{1}}, \bar{\nu}_{D}\right)
\end{array}\right), \quad U=\left(\begin{array}{ll}
\bar{\mu}_{a_{1}, 6 \rightarrow 7} & \bar{\nu}_{D, 6 \rightarrow 7}+\gamma_{6 \rightarrow 7}\left(-\mu_{a_{1}}, \bar{\nu}_{D}\right)
\end{array}\right) \\
& V=\left(\begin{array}{ll}
\bar{\delta}_{a_{2}, a_{3}, 3 \rightarrow 5}+\gamma_{3 \rightarrow 5}\left(-\mu_{a_{1}}, \bar{\delta}_{a_{2}, a_{3}}\right)+\gamma_{3 \rightarrow 5}\left(-\nu_{D}, \bar{\delta}_{a_{2}, a_{3}}+\gamma\left(-\mu_{a_{1}}, \bar{\delta}_{a_{2}, a_{3}}\right)\right)
\end{array}\right) \\
& W=\left(\begin{array}{l}
\bar{\delta}_{a_{2}, a_{3}, 6 \rightarrow 7}+\gamma_{6 \rightarrow 7}\left(-\mu_{a_{1}}, \bar{\delta}_{a_{2}, a_{3}}\right)+\gamma_{6 \rightarrow 7}\left(-\nu_{D}, \bar{\delta}_{a_{2}, a_{3}}+\gamma\left(-\mu_{a_{1}}, \bar{\delta}_{a_{2}, a_{3}}\right)\right)
\end{array}\right)
\end{aligned}
$$

We first determine conditions under which $T$ is of full rank. The rank of this matrix is equal to the rank of the matrix

$$
\bar{T}=\left(\begin{array}{c}
T_{1} \\
T_{2}+\mu_{a_{1}, 1} T_{1} \\
T_{3}+\mu_{a_{1}, 2} T_{1}
\end{array}\right)
$$

with $T_{i}$ denoting the $i$-th row of $T$. It follows from (46), (54), and (65) that

$$
\begin{aligned}
& \bar{T}=\left(\begin{array}{cc}
\bar{\mu}_{a_{1}, 3} & \bar{\nu}_{D, 3}-\mu_{a_{1}, 1} \bar{\nu}_{D, 2}+\mu_{a_{1}, 2} \bar{\nu}_{D, 1} \\
0 & \bar{\nu}_{D, 4}+\frac{1}{2} \mu_{a_{1}, 1}\left(-\mu_{a_{1}, 1} \bar{\nu}_{D, 2}+\mu_{a_{1}, 2} \bar{\nu}_{D, 1}\right) \\
0 & \bar{\nu}_{D, 5}+\frac{1}{2} \mu_{a_{1}, 2}\left(-\mu_{a_{1}, 1} \bar{\nu}_{D, 2}+\mu_{a_{1}, 2} \bar{\nu}_{D, 1}\right)
\end{array}\right) \\
& =\left(\begin{array}{cc}
\frac{a_{1,1} a_{1,2}}{2} & \bar{\nu}_{D, 3}-\mu_{a_{1}, 1} \bar{\nu}_{D, 2}+\mu_{a_{1}, 2} \bar{\nu}_{D, 1} \\
\left(\begin{array}{l}
0 \\
0
\end{array}\right) & \left.\frac{1}{2} P \bar{D} Q\left(S\left(e_{1}\right)^{2}-2 S\left(e_{3}\right)\right)+O(|D|)^{3}\right)+O(|D|) O\left(\left|a_{1}\right|^{2}\right)
\end{array}\right)
\end{aligned}
$$

where the last equality comes from (23), (54), and (57). Since $S\left(e_{1}\right)^{2}-2 S\left(e_{3}\right)$ is invertible, so that the rank of the matrix $P \bar{D} Q\left(S\left(e_{1}\right)^{2}-2 S\left(e_{3}\right)\right)$ is equal to two when $D$ is invertible, it follows from (23) that the rank of $\bar{T}$ is equal to three if, for example, $a_{1,1}=a_{1,2}=d_{1}=d_{2}=d_{3}>0$ is chosen small enough. 
Let us now consider the terms $V$ and $W$. Let $a_{2,1}=a_{3,1}=\kappa>0$ and $a_{2,1}=a_{3,1}=\kappa^{3}$. Then, it follows from (59) that

$$
\forall i, \quad\left|\bar{\delta}_{a_{2}, a_{3}, i}\right| \leq 2 \kappa^{\ell(i)}
$$

and

$$
\left(\begin{array}{l}
\bar{\delta}_{a_{2}, a_{3}, 6} \\
\bar{\delta}_{a_{2}, a_{3}, 7}
\end{array}\right)=\frac{1}{2} \kappa^{4} W_{0} \quad \text { with } \quad W_{0}=\left(\begin{array}{cc}
1+\frac{1}{12} \sin \theta_{3} \sin ^{2} \theta_{2} \cos \theta_{2} & -\frac{1}{12} \sin ^{3} \theta_{2} \cos \theta_{3} \\
\frac{1}{12} \sin ^{3} \theta_{3} \cos \theta_{2} & 1-\frac{1}{12} \sin \theta_{2} \sin ^{2} \theta_{3} \cos \theta_{3}
\end{array}\right)
$$

Now, Property 1 of Lemma 1 implies that $\gamma_{i}\left(-\mu_{a_{1}}, \bar{\delta}_{a_{2}, a_{3}}\right)$ is linear in $\bar{\delta}_{a_{2}, a_{3}}$ and only depends on the components $\bar{\delta}_{a_{2}, a_{3}, k}$ of $\bar{\delta}_{a_{2}, a_{3}}$ such that $\ell(k)<\ell(i)$. This implies, using (67), that $\left|\gamma_{i}\left(-\mu_{a_{1}}, \bar{\delta}_{a_{2}, a_{3}}\right)\right| \leq$ $c \kappa^{\ell(i)-1}$ with $c$ denoting some constant. From the above definition of $V$ and $W$ one then deduces that

$$
\kappa \geq 1 \Longrightarrow\left|V_{i}\right| \leq c_{i} \kappa^{\ell(i+2)} \leq c_{i} \kappa^{3} \quad \text { and } \quad W=\frac{1}{2} \kappa^{4} W_{0}+R, \quad|R| \leq c_{0} \kappa^{3}
$$

with $V_{i}$ the $i$-th row of $V$ and $c_{i}, c_{0}$ some constants. The possible dependence of these constants upon $a_{1}$ and $D$ is not important. Note also that the above expression implies that $W$ is invertible for $\kappa$ large enough, since $W_{0}$ is invertible.

Since $T$ is of full rank, there exist for any $\left(\theta_{1}, Q\right)$ three column vectors of $T$ which form a $3 \times 3$ invertible matrix. Without lost of generality, let us assume that these columns are the three first ones and consider the $4 \times 4$ submatrix $H_{0}^{-}$of $H_{0}$ obtained by omiting the fourth column of $H_{0}$. Then,

$$
H_{0}^{-}=\left(\begin{array}{cc}
T^{-} & V \\
U^{-} & W
\end{array}\right)
$$

with $T^{-}$invertible. Recall that $H_{0}^{-}$is invertible if and only if the Shur complement $W-U^{-}\left(T^{-}\right)^{-1} V$ is invertible. Since neither $T^{-}$nor $U^{-}$depend on $\kappa$, it follows from (68) that this property is satisfied for $\delta$ large enough. Therefore, for any $\theta_{1}, \theta_{2}, \theta_{3}, Q$ there exists $\kappa>0$ such that $H_{0}$ is of full rank. Due to the compacity of $\mathbb{T}^{3} \times \mathbb{S O}(3)$, this in turn implies the existence of $\kappa>0$ such that for any $\theta_{1}, \theta_{2}, \theta_{3}, Q$, the matrix $H_{0}$ is of full rank.

\section{Proof of Lemma 1}

For any $\varepsilon$ and any $v, w \in \mathbb{R}^{7}$,

$$
\begin{aligned}
{\left[X \Delta_{\varepsilon} v, X \Delta_{\varepsilon} v\right] } & =\left[\sum_{i=1}^{7} X_{i} \varepsilon^{\ell(i)} v_{i}, \sum_{j=1}^{7} X_{j} \varepsilon^{\ell(j)} w_{j}\right] \\
& =\sum_{i<j}\left[X_{i}, X_{j}\right] \varepsilon^{\ell(i)+\ell(j)}\left(v_{i} w_{j}-v_{j} w_{i}\right)
\end{aligned}
$$

If $\ell(i)+\ell(j)>\max _{k} \ell(k)=4$, then $\left[X_{i}, X_{j}\right] \varepsilon^{\ell(i)+\ell(j)}=X \Delta_{\varepsilon} O(\varepsilon)$ since $\max _{k} \Delta_{\varepsilon}(k, k)=4$ and $\left[X_{i}, X_{j}\right] \in$ $g=\operatorname{span}_{\mathbb{R}}\left\{X_{1}, \ldots, X_{7}\right\}$. Therefore,

$$
\left[X \Delta_{\varepsilon} v, X \Delta_{\varepsilon} w\right]=A_{1}+X \Delta_{\varepsilon} O(\varepsilon) \quad \text { with } \quad A_{1}=\sum_{\substack{i<j \\ \ell(i)+\ell(j) \leq 4}}\left[X_{i}, X_{j}\right] \varepsilon^{\ell(i)+\ell(j)}\left(v_{i} w_{j}-v_{j} w_{i}\right)
$$

The sum $A_{1}$ can be written as

$\mathrm{RR} \mathrm{n}^{\circ} 7938$

$$
\begin{aligned}
A_{1}= & {\left[X_{1}, X_{2}\right] \varepsilon^{2}\left(v_{1} w_{2}-v_{2} w_{1}\right)+\left[X_{1}, X_{3}\right] \varepsilon^{3}\left(v_{1} w_{3}-v_{3} w_{1}\right)+\left[X_{1}, X_{4}\right] \varepsilon^{4}\left(v_{1} w_{4}-v_{4} w_{1}\right) } \\
& +\left[X_{2}, X_{3}\right] \varepsilon^{3}\left(v_{2} w_{3}-v_{3} w_{2}\right)+\left[X_{2}, X_{5}\right] \varepsilon^{4}\left(v_{2} w_{5}-v_{5} w_{2}\right) \\
& +\left[X_{1}, X_{5}\right] \varepsilon^{4}\left(v_{1} w_{5}-v_{5} w_{1}\right)+\left[X_{2}, X_{4}\right] \varepsilon^{4}\left(v_{2} w_{4}-v_{4} w_{2}\right) \\
= & X_{3} \varepsilon^{2}\left(v_{1} w_{2}-v_{2} w_{1}\right)+X_{4} \varepsilon^{3}\left(v_{1} w_{3}-v_{3} w_{1}\right)+X_{6} \varepsilon^{4}\left(v_{1} w_{4}-v_{4} w_{1}\right) \\
& +X_{5} \varepsilon^{3}\left(v_{2} w_{3}-v_{3} w_{2}\right)+X_{7} \varepsilon^{4}\left(v_{2} w_{5}-v_{5} w_{2}\right) \\
& +X \Delta_{\varepsilon} O(\varepsilon)
\end{aligned}
$$


where the last equality comes from the definition (26) of the $X_{i}$ 's and from (25) which allows one to express $\left[X_{1}, X_{5}\right]$ and $\left[X_{2}, X_{4}\right]$ as a linear combination of $X_{1}, \ldots, X_{5}$. Therefore,

$$
\left[X \Delta_{\varepsilon} v, X \Delta_{\varepsilon} v\right]=X \Delta_{\varepsilon}\left[\beta(v, w)+O(\varepsilon], \quad \beta(v, w)=\left(\begin{array}{c}
0_{2 \times 1} \\
v_{1} w_{2}-v_{2} w_{1} \\
v_{1} w_{3}-v_{3} w_{1} \\
v_{2} w_{3}-v_{3} w_{2} \\
v_{1} w_{4}-v_{4} w_{1} \\
v_{2} w_{5}-v_{5} w_{2}
\end{array}\right)\right.
$$

and (49) follows.

Let us now establish (50). From the above equation,

$$
\begin{aligned}
& \operatorname{ad}^{2}\left(X \Delta_{\varepsilon} v\right)\left(X \Delta_{\varepsilon} w\right)=\left[X \Delta_{\varepsilon} v, \operatorname{ad}^{1}\left(X \Delta_{\varepsilon} v\right)\left(X \Delta_{\varepsilon} w\right)\right] \\
& =\left[\sum_{i=1}^{7} X_{i} \varepsilon^{\ell(i)} v_{i}, \sum_{j=1}^{7} X_{j} \varepsilon^{\ell(j)}\left(\beta_{j}(v, w)+O(\varepsilon)\right)\right] \\
& =\left[\sum_{i=1}^{7} X_{i} \varepsilon^{\ell(i)} v_{i}, \sum_{j=1}^{7} X_{j} \varepsilon^{\ell(j)} \beta_{j}(v, w)\right]+X \Delta_{\varepsilon} O(\varepsilon) \\
& =A_{2}+X \Delta_{\varepsilon} O(\varepsilon), \quad A_{2}=\sum_{i<j}\left[X_{i}, X_{j}\right] \varepsilon^{\ell(i)+\ell(j)}\left(v_{i} \beta_{j}(v, w)-v_{j} \beta_{i}(v, w)\right) \\
& \ell(i)+\ell(j) \leq 4
\end{aligned}
$$

By using (26), (25), and the fact that $\beta_{1}=\beta_{2}=0$, one obtains

$$
\begin{aligned}
A_{2}= & {\left[X_{1}, X_{3}\right] \varepsilon^{3} v_{1} \beta_{3}(v, w)+\left[X_{1}, X_{4}\right] \varepsilon^{4} v_{1} \beta_{4}(v, w) } \\
& +\left[X_{2}, X_{3}\right] \varepsilon^{3} v_{2} \beta_{3}(v, w)+\left[X_{2}, X_{5}\right] \varepsilon^{4} v_{2} \beta_{5}(v, w) \\
& +\left[X_{1}, X_{5}\right] \varepsilon^{4} v_{1} \beta_{5}(v, w)+\left[X_{2}, X_{4}\right] \varepsilon^{4} v_{2} \beta_{4}(v, w) \\
= & X_{4} \varepsilon^{3} v_{1} \beta_{3}(v, w)+X_{6} \varepsilon^{4} v_{1} \beta_{4}(v, w)+X_{5} \varepsilon^{3} v_{2} \beta_{3}(v, w)+X_{7} \varepsilon^{4} v_{2} \beta_{5}(v, w)+X \Delta_{\varepsilon} O(\varepsilon)
\end{aligned}
$$

Eq. (50) directly follows from the above equalities.

The calculation of $\operatorname{ad}^{3}\left(X \Delta_{\varepsilon} v\right)\left(X \Delta_{\varepsilon} w\right)$ is similar and not reproduced here. Finally, for $k \geq 4$,

$$
\operatorname{ad}^{k}\left(X \Delta_{\varepsilon} v\right)\left(X \Delta_{\varepsilon} w\right)=O\left(\varepsilon^{k+1}\right)=O\left(\varepsilon^{5}\right)
$$

because $X \Delta_{\varepsilon} u=O(\varepsilon)$ for any $u$. Relation (52) follows by decomposing $\operatorname{ad}^{k}\left(X \Delta_{\varepsilon} v\right)\left(X \Delta_{\varepsilon} w\right)$ on the generating family of v.f. $X_{1}, \ldots, X_{6}, X_{7}$ of $\mathfrak{g}$, and using the fact that $\max _{k} \Delta_{\varepsilon}(k, k)=4$.

\section{Proof of Lemma 2;}

Recall the classical property (see e.g. [16])

$$
\operatorname{Ad}(\exp (Z)) X(e)=\exp (\operatorname{ad}(Z))(X)(e)=\sum_{k=0}^{+\infty} \frac{1}{k !} \operatorname{ad}^{k}(Z)(X)(e)
$$

Therefore,

$$
\operatorname{Ad}\left(\exp \left(X \Delta_{\varepsilon} v\right)\right) X(e) \Delta_{\varepsilon} w=\sum_{k=0}^{+\infty} \frac{1}{k !} \operatorname{ad}^{k}\left(X \Delta_{\varepsilon} v\right)\left(X \Delta_{\varepsilon} w\right)(e)
$$

From here, the proof easily follows by using the expressions of $\operatorname{ad}^{k}\left(X \Delta_{\varepsilon} v\right)\left(X \Delta_{\varepsilon} w\right)$ given in Lemma 1 


\section{Appendix 2: Proof of Proposition 2}

One deduces from (28) that the linearized system along a reference trajectory with constant curvature $c_{r}$ is given by

$$
\left\{\begin{array}{l}
\dot{\tilde{g}}_{1}=\tilde{v}_{1}+v_{r, 3} \tilde{g}_{2} \\
\dot{\tilde{g}}_{2}=v_{r, 1} \tilde{g}_{3}-v_{r, 3} \tilde{g}_{1} \\
\dot{\tilde{g}}_{3}=\tilde{v}_{2} \\
\dot{\tilde{\eta}}_{1}=v_{r, 1} \tilde{\eta}_{1}+c_{r} \tilde{v}_{1}-\tilde{v}_{2} \\
\dot{\tilde{\eta}}_{2}=-v_{r, 1} \tilde{\eta}_{2}-c_{r} \tilde{v}_{1}+\tilde{v}_{2}
\end{array}\right.
$$

with $\tilde{\eta}=\eta-\eta_{r}$. By using the change of variables (41) one obtains the following equivalent system

$$
\left\{\begin{array}{l}
\dot{x}_{1}=\tilde{v}_{1}+v_{r, 3} x_{2} \\
\dot{x}_{2}=v_{r, 1} x_{3}-v_{r, 3} x_{1} \\
\dot{x}_{3}=\tilde{v}_{2} \\
\dot{x}_{4}=v_{r, 1} x_{5}-v_{r, 1} x_{2}+c_{r} \tilde{v}_{1} \\
\dot{x}_{5}=v_{r, 1} x_{4}-v_{r, 3} x_{1}
\end{array}\right.
$$

Setting $\tilde{v}_{1}=-v_{r, 3} x_{2}-k_{4}\left|v_{r, 1}\right| x_{1}$ with $k_{4}>0$ ensures the exponential stability of $x_{1}=0$ for any $v_{r, 1}$ that satisfies (43). The zero-dynamics associated with $x_{1}=0$ is

$$
\left\{\begin{array}{l}
\dot{x}_{2}=v_{r, 1} x_{3} \\
\dot{x}_{3}=v_{2} \\
\dot{x}_{4}=v_{r, 1} x_{5}-\left(1+c_{r}^{2}\right) v_{r, 1} x_{2} \\
\dot{x}_{5}=v_{r, 1} x_{4}
\end{array}\right.
$$

Let us first assume that $v_{r, 1}=1$. Then, $x_{5}^{(4)}=x_{5}^{(2)}-\left(1+c_{r}^{2}\right) \tilde{v}_{2}$ and an exponential stabilizer of the origin of the above system is thus given by

$$
\tilde{v}_{2}=\frac{1}{1+c_{r}^{2}}\left(k_{3} \dddot{x}_{5}+\left(1+k_{2}\right) \ddot{x}_{5}+k_{1} \dot{x}_{5}+k_{0} x_{5}\right)
$$

with $k_{0,1,2,3}$ any set of gain values that make the polynomial $s^{4}+k_{3} s^{3}+k_{2} s^{2}+k_{1} s+k_{0}$ Hurwitz-stable. By introducing the time-scaling $\tau=\int_{0}^{t}\left|v_{r, 1}(\alpha)\right| d \alpha$ one then deduces from (72) that $\tilde{v}_{2}$ given by (42) is an exponential stabilizer of the origin of System (71) for any $v_{r, 1}$ that satisfies (43). Since $x_{1}=0$ is also exponentially stable under the same condition, the origin of the complete linearized system (70) is exponentially stable. Now, since the tracking-error dynamics is affine w.r.t. $v_{r}$, the original (non-linear) system can be written as

$$
\dot{x}=A\left(v_{r}\right) x+R(x) v_{r}
$$

with $R(0)=0, \frac{\partial R}{\partial x}(0)=0$, and the origin of the linearized system $\dot{x}=A\left(v_{r}\right) x$ exponentially stable. Local exponential stability of the origin of this system follows from the assumption that $v_{r}$ is bounded and from classical exponential stability results [17, Th. 4.13]. 
informatics

RESEARCH CENTRE

SOPHIA ANTIPOLIS - MÉDITERRANÉE

2004 route des Lucioles - BP 93

06902 Sophia Antipolis Cedex
Publisher

Inria

Domaine de Voluceau - Rocquencourt

BP 105 - 78153 Le Chesnay Cedex

inria.fr

ISSN 0249-6399 